\title{
Article \\ An SVEIRE Model of Tuberculosis to Assess the Effect of an Imperfect Vaccine and Other Exogenous Factors
}

\author{
Fatima Sulayman ${ }^{*}{ }^{\dagger}$, Farah Aini Abdullah ${ }^{+}$and Mohd Hafiz Mohd ${ }^{\dagger}$ \\ School of Mathematical Sciences, Universiti Sains Malaysia (USM), Penang 11800, Malaysia; \\ farahaini@usm.my (F.A.A.); mohdhafizmohd@usm.my (M.H.M.) \\ * Correspondence: fatisulay@student.usm.my \\ t These authors contributed equally to this work.
}

check for

updates

Citation: Sulayman, F.; Abdullah,

F.A.; Mohd, M.H. An SVEIRE Model

of Tuberculosis to Assess the Effect of

an Imperfect Vaccine and Other

Exogenous Factors. Mathematics 2021,

9, 327. https://doi.org/

$10.3390 /$ math 9040327

Academic Editor: Ricardo Lopez-Ruiz Received: 2 November 2020

Accepted: 5 January 2021

Published: 7 February 2021

Publisher's Note: MDPI stays neutral with regard to jurisdictional clai$\mathrm{ms}$ in published maps and institutional affiliations.

Copyright: $\odot 2021$ by the authors. Licensee MDPI, Basel, Switzerland. This article is an open access article distributed under the terms and conditions of the Creative Commons Attribution (CC BY) license (https:// creativecommons.org/licenses/by/ $4.0 /)$.

\begin{abstract}
This study extends a deterministic mathematical model for the dynamics of tuberculosis transmission to examine the impact of an imperfect vaccine and other exogenous factors, such as re-infection among treated individuals and exogenous re-infection. The qualitative behaviors of the model are investigated, covering many distinct aspects of the transmission of the disease. The proposed model is observed to show a backward bifurcation, even when $R v<1$. As such, we assume that diminishing $R v$ to less than unity is not effective for the elimination of tuberculosis. Furthermore, the results reveal that an imperfect tuberculosis vaccine is always effective at reducing the spread of infectious diseases within the population, though the general effect increases with the increase in effectiveness and coverage. In particular, it is shown that a limited portion of people being vaccinated at steady-state and vaccine efficacy assume a equivalent role in decreasing disease burden. From the numerical simulation, it is shown that using an imperfect vaccine lead to effective control of tuberculosis in a population, provided that the efficacy of the vaccine and its coverage are reasonably high.
\end{abstract}

Keywords: tuberculosis model; vaccination; effective reproduction number; stability analysis; bifurcation analysis

\section{Introduction}

Tuberculosis (TB) is a communicable disease that affects the lungs; however, it can also attack different organs, including the brain, kidney, spine, central nervous system, and the lymphatic system [1-3]. Some studies have suggested that up to a third of the global human population has contracted the disease [4]. Tuberculosis is caused by a family of bacteria called Mycobacterium tuberculosis (Mtb) [1]. This airborne infectious disease is a public health challenge worldwide: In the USA [5], European nations [6,7], and developingcountries [1,8]. In other words, tuberculosis remains a major epidemic that affects the morbidity and mortality [1,9-11] of both human and animal populations [12]. Usually, when the disease is contracted by individuals, it is not immediately obvious; for instance, when a person is infected, he or she may remain infected for years, or may even remain latently infected for life [13]. It has been attested to be highly prevalent in middle-income countries, among other regions [1]. The global prevalence of TB in 2018 was about 1 million cases more than that of HIV / AIDS, with up to 1.5 million more deaths [1].

TB can be transmitted, either through direct or indirect contact with an infected person $[7,14]$. The symptoms of tuberculosis include a constant cough that produces blood and/or sputum (phlegm from deep inside the lungs), chest pain, fever, and sweating at night, among others [1].

It has been discovered, through clinical observation, that variable rates of progression to active TB after infection exist [15]. Proof from an interferon-gamma release assay has suggested that over about 12.9 percent of infected individuals progress to active TB within the first 23 months after infection [16]. In addition, after the high-risk time frame, the rate 
at which active $\mathrm{TB}$ happens is moderately low within a few years, ranging from $5 \%$ to $10 \%$ [17-20]. The long latent period of MTb bacteria prolongs the start of the active phase of the disease, thus making it difficult to understand how the disease develops. Already infected Individuals (even in the latent stage) whose level of immunity is low may become newly infected through contact with an infectious individual [21]. Therefore, anyone in the latent phase of TB can move to the active phase in two ways: Due to exogenous re-infection or endogenous reactivation, known as "slow progression" (see [17,21-23]).

Generally, three kind of medical interventions are available for the treatment of TB: Treating latently infected TB individuals to prevent endogenous reactivation; treatment to cure active TB patients; and vaccination to reduce TB epidemics [24]. For some infections (pertussis, mumps, tuberculosis, and so on), the vaccination of susceptible individuals is a viable strategy for controlling the epidemic spread $[25,26]$. At the point when it is included in mathematical compartmental models, vaccination is frequently represented by a direct exchange between the susceptible individuals and the recovered compartment [27]. Some vaccinations prevent the chance of infection but do not prevent the vaccinated individual from securing and spreading the infection [28].

At present, the main successful TB vaccine in common use is Bacille Calmette-Guerin (BCG) [29], which is efficient in children and has greater than 50\% insurance against lung infection and over $80 \%$ of types of TB. Most significantly, children do not spread TB, while adults do [30]. Unfortunately, BCG has shown variable-mostly poor-protection against TB in adults. As such, new vaccines that target both children and adult populations are needed [31].

The role of exogenous factors, such as exogenous re-infection and re-infection between treated individuals, and imperfect vaccines in TB epidemics have been examined by several authors using mathematical models (see, e.g., [2,19,21,22,26,32-35]). Bhunu et al. [33] studied a more broad TB model including exogenous re-infection.

Over the past few decades, a small number of SEIR models incorporating vaccination compartments have been extensively formulated and presented from a mathematical point of view in the litertaure, in order to evaluate the potential effect of an imperfect vaccine on mitigating the transmission of TB [36-40]. A particular result frequently arising from analyses of imperfect vaccinations and other exogenous factors of TB epidemic models is the phenomenon of backward bifurcation: The circumstance where reducing the basic reproduction number below 1 is not enough to eradicate the epidemic [21,22,28,34].

Generally, these classical epidemic models have just a single endemic equilibrium when the basic reproduction number $R_{0}>1$. The stability of the disease-free equilibrium is ensured when $R_{0}<1$ and unstable when $R_{0}>1$. Hence, the bifurcation deriving from the disease-free equilibrium to an endemic equilibrium is forward. However, as of recent years, the backward bifurcation phenomenon has emerged in research on infection control (see $[28,38,41])$. In this situation, the basic reproduction number cannot accurately illustrate the important disease eradication any longer. Accordingly, it is essential to identify backward bifurcations and set up thresholds for the control of infections.

The backward bifurcation phenomenon offers an alternate interpretation, as it demonstrates that, even though $R_{0}<1$ and the DFE is steady, another steady endemic equilibrium can still coexist simultaneously $[28,41]$. Therefore, even though $R_{0}<1$, a population could be at an endemic equilibrium wherein the infection persists indefinitely [41]. When several stable equilibria coexist simultaneously, the final equilibrium that a population will reach relies upon the initial conditions (i.e., regarding the number of people) of its subpopulations [41].

The epidemiological significance of the backward bifurcation phenomena has been extensively examined in the literature $[21,22,38]$. Regarding epidemiological models, the phenomena was first investigated in an HIV model that separated the sexually active population into $n$ mixing groups of various risk levels [42]. However, this kind of phenomenon may emerge due to epidemiological mechanisms that are related to the use of imperfect intervention techniques [28,38,43-45]; relating to incomplete immunity [43,46,47], behavioral 
responses [42,43,48], epidemic models that account for immunological conditions [49,50], inadequate treatment resources [51-53], cohort structured epidemic models for fatal diseases or fatal infections [54,55], vertical transmission and non-linear incidence [56], infection-age structuring [57], and coinfection [58].

Although vaccination is just one of the mitigation strategies against communicable diseases, mass vaccination has been distinguished as an effective disease prevention method. Recently, it was realized that even though many susceptible people have been vaccinated, costing a lot of money, the spread of infectious diseases is yet to be contained. As they are still endemic in virtually all countries of the world. Vaccines are biologically required to inspire an invulnerable reaction, similar to that which would be set off by a characteristic infection without causing the genuine, infectious disease [59]. This kind of vaccine, that partially prevents infection spread by permitting restricted transmission of a pathogen, disease, or virus is called a leaky or imperfect vaccine. According to the investigation of [59], there are three kinds of imperfect vaccines: The first is known as a leaky vaccine, whereby vaccination diminishes infection; however, it does not eradicate the chance of infection after exposure to an infectious disease. The second is the all-or-nothing vaccine, which provides specific individuals with lifetime immunity; however, it provides no protection for others. The third vaccine is waning, which turns out to be effective only for a brief period. An "imperfect vaccine" is a vaccine that is unable to protect all immunized susceptible individuals [60]. We consider it important to understand the influence of imperfect vaccines, given the existing literature, which guaranteed their incorporation in the proposed model; in addition, only a few researchers have studied them [38-40] in the mathematical modeling of TB.

This study was inspired by a paper developed by Kar and Mondal [21], in which they proposed a TB model with SEIT components, later reduced to SEI components. The current study permits that susceptible people might receive an imperfect vaccine to reduce their vulnerability to tuberculosis. Thus, the class $\mathrm{V}$ of vaccinated people is considered as a susceptible compartment. However, we also incorporate an imperfect vaccine that wanes with time and with efficacy less than $100 \%$ [61] between these two compartments, making the model different from that of Kar and Mondal [21].

This paper is arranged as follows: In Section 2, the model is formulated. Its basic properties are derived in Section 3. Section 4 is devoted to the stability of disease-free and effective basic reproduction number. The stability of endemic equilibrium and bifurcation analysis is detailed in Section 5. Numerical simulations are presented in Section 6. Finally, our conclusions and discussions are presented in Sections 7 and 8.

\section{Model and Methods}

In this section, we employ a non-linear ODE system of S-V-E-I-R type, which is a modification of the model developed by Kar and Mondal [21]. The whole population $N(\mathrm{t})$ is composed of five (5) compartments, $S, V, E, I$, and $R$, which respectively denote susceptible, vaccinated, exposed, infected, and recovered individuals. Kar and Mondal [21] assumed that individuals that had been treated can be re-infected if their immunity is low. This leads to the S-V-E-I-R-E type system for modelling the transmission dynamics of TB. The S-V-E-I-R-E process of TB spreading is shown in Figure 1.

\section{Biological Assumptions of the Model}

The biological assumptions of the model are as follows

- There is a constant recruitment rate to the susceptible population and natural cause death affects individuals in all compartments, with an extra TB-induced death rate in the infected class;

- Some susceptible individuals who have been successfully vaccinated lose their vaccineinduced immunity (i.e., vaccine failed), and these previously vaccinated individuals join the susceptible compartment again [38]. 
- Those in the vaccination class are not at a completely protective level, due to the imperfect vaccine. The vaccinated individuals become infected and move into the exposed class [40]. We assume that this occurs at a lower transmission rate $\omega \beta$, where $\omega \in[0,1]$ is the decrease coefficient [39].

- $\quad$ The individuals in the susceptible class move to the exposed class with the transmission rate $\beta$.

- The individuals in the exposed class become infectious and move to the infected class. After recovery, they move to the recovered compartment. When the recovered individuals loose immunity, they return to the exposed class [21].

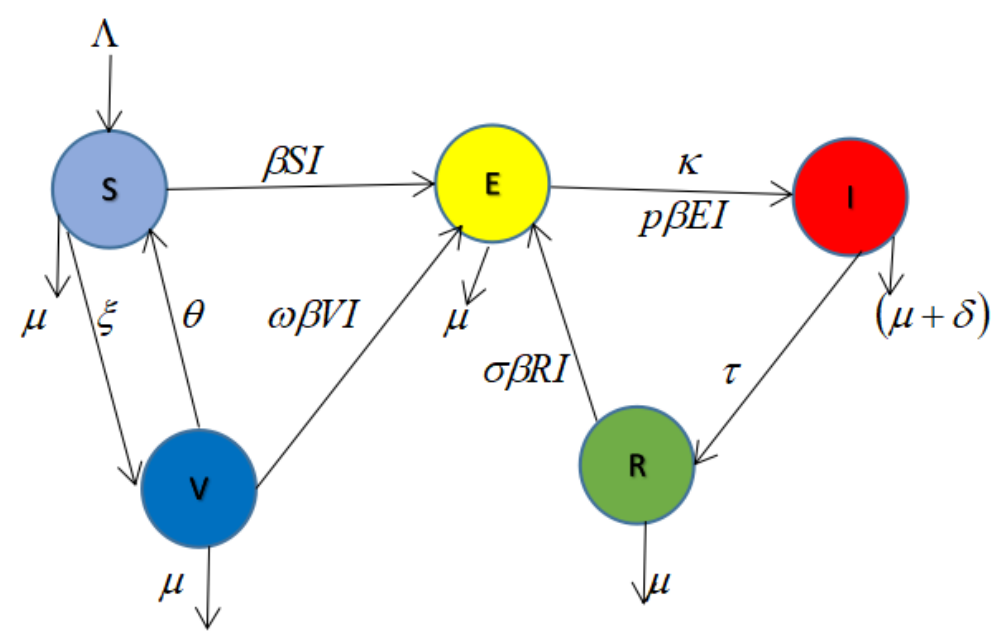

Figure 1. The schematic diagram of the SVEIRE TB model (see Equation (1)).

In this epidemiological system, the susceptible compartment is increased through the recruitment of individuals, either by immigration or birth into the population at a constant rate $\Lambda$, while each compartment is diminished at the rate $\mu$. Administration of vaccines to the susceptible population moves individuals to the vaccinated compartment at the rate $\xi$; however, the protection provided by the vaccine wanes over time, at the rate of $\theta$. The susceptible individuals become infected at a rate $\beta S I$, known as mass action. The vaccinated compartment individuals acquire infection through contact with exposed individuals. As the vaccine offered to individuals is thought to be imperfect (i.e., it does not offer $100 \%$ assurance against the TB disease), the vaccinated individuals may become infected again; but with a lower level than those in the susceptible compartment (i.e., unvaccinated individuals). Therefore, the transmission rate $\beta$ is estimated by a scaling factor $(1-\omega)$, where $0 \leq \omega \leq 1$ is the viability of the vaccine: $\omega=1$ implies that a vaccine provides $100 \%$ assurance against the TB disease, while $\omega=0$ indicates a vaccine that does not secure individuals in any way $[60,62]$. The exposed compartment becomes infectious at the rate of $\kappa$ E and progresses to actively infected state. Exogenous re-infection can enable individuals that are already infected to develop active tuberculosis. This happens when they contract new infection from infectious individuals at a constant rate $p \beta E I$. Infected individuals are recovered with the rate $\tau I$ and decreased due to TB-induced death at a rate $\delta$. Finally, the recovered individuals may return to the exposed compartment, due to low immunity, with rate $\sigma$. 


$$
\begin{aligned}
& \frac{d S}{d t}=\Lambda+\theta V-\xi S-\beta S I-\mu S \\
& \frac{d V}{d t}=\xi S-\omega \beta V I-\theta V-\mu V \\
& \frac{d E}{d t}=\beta S I+\omega \beta V I-p \beta E I-(k+\mu) E+\sigma \beta I R \\
& \frac{d I}{d t}=p \beta E I+k E-(\mu+\tau+\delta) I \\
& \frac{d R}{d t}=\tau I-\sigma \beta I R-\mu R,
\end{aligned}
$$

with nonnegative initial conditions given by

$$
S(0)=S_{0}>0, V(0)=V_{0}>0, E(0)=E_{0}>0, I(0)=I_{0}>0, R(0)=R_{0}>0 .
$$

All the parameters of system (1) are assumed to be positive for all time $t>0$.

\section{Mathematical Model Analysis}

\subsection{Positivity of Solutions}

For the tuberculosis infection model system (1) to be epidemiologically realistic, it is necessary to prove that all the state variables remain positive for all time.

Theorem 1. Let the initial data be $\{(S, V, E, I, R) \geq 0\} \in \Phi$. Then, the solution set $\{S(t), V(t), E(t), I(t), R(t)\}$ of the model system (1) is non-negative for all $t>0$.

Proof. As mentioned in the study of Obasi and Mbah [63], considering the non-linear system of model (1), we take the first equation

$$
\frac{d S}{d t}=\Lambda-\beta S I-\mu S
$$

that is,

$$
\frac{d S}{d t} \geq-(\beta I+\mu) S
$$

Integrating (3), by separation of variables, gives

$$
\begin{aligned}
& \int \frac{d S}{S} \geq \int-(\beta I+\mu) d t . \\
& \ln S \geq-(\beta I+\mu) t \\
& \log _{e} S \geq-(\beta I+\mu) t \\
& S \geq e^{-(\beta I+\mu) t} \\
& \Rightarrow \\
& S(t) \geq S(0) e^{-\mu t-\beta \int I d t} \\
& S(t) \geq S(0) e^{(-\mu t-\beta I t)} \\
& S(t) \geq 0 .
\end{aligned}
$$




\subsection{Invariant Region}

Theorem 2. For the initial conditions (2), the solutions of system (1) are contained in the region $\Phi \subset R_{+}^{5}$, defined by

$$
\Phi=\left[(S(t), V(t), E(t), I(t), R(t)) \in R_{+}^{5}: N(t) \leq \frac{\Lambda}{\mu}\right] .
$$

Proof. Summation of all equations of model system (1) gives

$$
\frac{d N(t)}{d t}=\frac{d S(t)}{d t}+\frac{d V(t)}{d t}+\frac{d E(t)}{d t}+\frac{d I(t)}{d t}+\frac{d R(t)}{d t} .
$$

The change in the complete population is defined by

$$
\frac{d N}{d t}=\Lambda-\mu N-\delta I(t)
$$

which gives

$$
\frac{d N}{d t} \leq \Lambda-\mu N
$$

The solution of Equation (9) is given by

$$
N(t) \leq \frac{\Lambda}{\mu}-\left(\frac{\Lambda}{\mu}-N_{0}\right) e^{-\mu t},
$$

where $N_{0}=N(0)$.

Using the Birkhoff-Rota theorem [67], we note that, if $N_{0}<\frac{\Lambda}{\mu}$, then $N \rightarrow \frac{\Lambda}{\mu}$ asymptotically as $\mathrm{t} \rightarrow \infty$ in Equation (6) and the total population size $N \rightarrow \frac{\Lambda}{\mu}$, which means that $0 \leq N$ $\leq \frac{\Lambda}{\mu}$. Therefore, all the feasible solutions in the model converge in the region $\Phi$ [68].

\section{Analysis of Disease-Free Equilibrium (DFE), $P_{0}$, and Basic Reproduction Number $R_{0}$}

The disease-free equilibrium (DFE) state, $P_{0}$, is a steady-state solution where there is no infection in the community. The disease class can be described as the infected human population. Taking the first equation of (1) with $E=I=R=0$ into consideration, we arrive at:

$$
S_{0}=\frac{(\mu+\theta) \Lambda}{\mu(\xi+\mu+\theta)} \text { and } V_{0}=\frac{\Lambda(\xi)}{\mu(\xi+\mu+\theta)} .
$$

Then, the disease-free equilibrium (DFE) state $P_{0}$ is given by

$$
P_{0}=\left[\frac{(\mu+\theta) \Lambda}{\mu(\xi+\mu+\theta)}, \frac{\Lambda(\xi)}{\mu(\xi+\mu+\theta)}, 0,0,0\right]
$$

\subsection{Basic Reproduction Number $\left(R_{0}\right)$}

Diekmann et al. [69] and Dietz [70] defined the basic reproduction number, $R_{0}$, as the effective number of secondary infections caused by a primary infected individual during their entire period of infectiousness. To obtain the basic reproduction number, we used the next generation matrix method of [71], where $F$ is the matrix of the new infection terms and $V$ is the matrix of the transition terms. The matrices $F$ and $V$ can be established from the 
coefficients of $E$ and $I$ in the second and last equations of system (1). The model equations are re-written, starting with newly infective classes:

$$
\begin{gathered}
\frac{d E}{d t}=\beta S I+\omega \beta V I-p \beta E I-(\mu+\kappa) E+\sigma \beta I R, \\
\frac{d I}{d t}=p \beta E I+k E-(\mu+\tau+\delta) I \\
F=\left[\begin{array}{ccc}
0 & \frac{\omega \beta \Lambda \xi}{\mu(\xi+\mu+\theta)}+\frac{\beta(\mu+\theta) \Lambda}{\mu(\xi+\mu+\theta)} & 0 \\
0 & 0 & 0 \\
0 & 0 & 0
\end{array}\right] \text { and } V=\left[\begin{array}{ccc}
(\mu+\kappa) & 0 & 0 \\
-\kappa & (\mu+\tau+\delta) & 0 \\
0 & -\tau & \mu .
\end{array}\right]
\end{gathered}
$$

The basic reproduction number is the spectral radius of $F V^{-1}[69,71]$, which must be considered when analyzing any epidemiological model [71]. Thus, the effective reproduction number for this epidemiological system is:

$$
R v=\frac{\Lambda \beta(\xi \omega+\mu+\theta) \kappa}{\mu(\xi+\mu+\theta)(\mu+\kappa)(\mu+\tau+\delta)} .
$$

The threshold quantity $(R v)$ is known as the control reproduction number of an epidemics; see Gumel et al. [60]. It aims to measure the estimated number of new TB cases generated by an index case (a single infected individual in a totally susceptible population) in a community with a vaccination programme set up [60]. Similarly, the basic reproduction number $R_{0}$ is accomplished by setting the parameters $\xi$ and $\theta=0$ in Equation (15), giving

$$
R_{0}=\frac{\Lambda \beta \kappa}{\mu(\mu+\kappa)(\mu+\tau+\delta)} .
$$

\subsection{Proving the Local Stability of Disease-Free Equilibrium Point}

Theorem 3. The disease-free equilibrium state, $P_{0}$, of the model system (1) is LAS when $R v<1$ and unstable if $R v>1$.

Proof. Consider the model system (1)

$$
f_{1}=\frac{d S}{d t}, f_{2}=\frac{d V}{d t}, f_{3}=\frac{d E}{d t}, f_{4}=\frac{d I}{d t}, f_{5}=\frac{d R}{d t} .
$$

The Jacobian of the system (1) is given by:

$$
\Sigma\left(P_{0}\right)=\left(\begin{array}{lllll}
\frac{\partial f_{1}}{\partial S} & \frac{\partial f_{1}}{\partial V} & \frac{\partial f_{1}}{\partial E} & \frac{\partial f_{1}}{\partial I} & \frac{\partial f_{1}}{\partial R} \\
\frac{\partial f_{2}}{\partial S} & \frac{\partial f_{2}}{\partial V} & \frac{\partial f_{2}}{\partial E} & \frac{\partial f_{2}}{\partial I} & \frac{\partial f_{2}}{\partial R} \\
\frac{\partial f_{3}}{\partial S} & \frac{\partial f_{3}}{\partial V} & \frac{\partial f_{3}}{\partial E} & \frac{\partial f_{3}}{\partial I} & \frac{\partial f_{3}}{\partial R} \\
\frac{\partial f_{4}}{\partial S} & \frac{\partial f_{4}}{\partial V} & \frac{\partial f_{4}}{\partial E} & \frac{\partial f_{4}}{\partial I} & \frac{\partial f_{4}}{\partial R} \\
\frac{\partial f_{5}}{\partial S} & \frac{\partial f_{5}}{\partial V} & \frac{\partial f_{5}}{\partial E} & \frac{\partial f_{5}}{\partial I} & \frac{\partial f_{5}}{\partial R}
\end{array}\right) .
$$

At the equilibrium point $\left(P_{0}\right)$, the Jacobian becomes:

$$
\left(\begin{array}{ccccc}
-(\mu+\xi) & \theta & 0 & -\beta(\theta+\mu) C_{4} & 0 \\
\xi & -(\mu+\theta) & 0 & -\omega \xi C_{4} & 0 \\
0 & 0 & -(\kappa+\mu) & C_{5} & 0 \\
0 & 0 & \kappa & -(\mu+\tau+\delta) & 0 \\
0 & 0 & 0 & \tau & -\mu
\end{array}\right),
$$

where 


$$
C_{4}=\frac{\Lambda}{\mu(\xi+\mu+\theta)}
$$

and

$$
C_{5}=\frac{\Lambda(\omega \xi+\mu+\theta)}{\mu(\xi+\mu+\theta)} .
$$

Three eigenvalues of (13) are $\lambda_{1}=-\mu, \lambda_{2}=-(\theta+\mu)$, and $\lambda_{3}=-(\xi+\mu)$, while the remaining two eigenvalues are obtained from the 2 by 2 matrix

$$
A=\left(\begin{array}{cc}
-(\kappa+\mu) & C_{5} \\
\kappa & -(\mu+\tau+\delta)
\end{array}\right) .
$$

Then, the eigenvalues of $\mathrm{A}$ are real and negative if the Routh-Hurwitz condition is satisfied. Applying the Routh-Hurwitz condition:

(i) $\operatorname{Tr}(\mathrm{A})<0$

(ii) $\operatorname{Det}(\mathrm{A})>0$.

Then,

$$
\begin{aligned}
\operatorname{Tr}(A) & =-(\kappa+\mu)-(\mu+\tau+\delta) \\
& =-(\kappa+2 \mu+\tau+\delta)<0 \\
\operatorname{Det}(A) & =(\kappa+\mu)(\mu+\tau+\delta)-\kappa C_{5} \\
& =(\kappa+\mu)(\mu+\tau+\delta)\left[1-\frac{\kappa C_{5}}{(\kappa+\mu)(\mu+\tau+\delta)}\right] \\
=(\kappa+\mu)(\mu+\tau+\delta)\left[1-\frac{\kappa \beta \Lambda(\omega \xi+\mu+\theta)}{\mu(\kappa+\mu)(\mu+\tau+\delta)(\xi+\mu+\theta)}\right] & (\kappa+\mu)(\mu+\tau+\delta)(1-R v)>0 \text { if } R v<1 .
\end{aligned}
$$

Following Theorem 2 of [71], we conclude that the DFE point is locally asymptotically stable.

\section{Endemic Equilibrium and Bifurcation Analysis}

The endemic equilibrium of the model system (1) is denoted by $P^{*}=\left(S^{*}, V^{*}, E^{*}, I^{*} R^{*}\right)$ and is determined by setting the right-hand side of model system one to zero:

$$
\begin{aligned}
& \Lambda+\theta V-\xi S-\beta S I-\mu S=0 \\
& \xi S-\omega \beta V I-\theta V-\mu V=0 \\
& \beta S I+\omega \beta V I-p \beta E I-(\kappa+\mu) E+\sigma \beta I R=0 \\
& p \beta E I+\kappa E-(\mu+\tau+\delta) I=0 \\
& \tau I-\sigma \beta I R-\mu R=0 .
\end{aligned}
$$

The first and second equations in (20) give

$$
\begin{aligned}
S^{*} & =\frac{\Lambda(\omega \beta I+\mu+\theta)}{\omega \beta^{2} I^{2}+\omega \beta^{2} I^{2}+G_{0} \beta I+G_{1}}, \\
V^{*} & =\frac{\Lambda \xi}{\omega \beta^{2} I^{2}+\omega \beta^{2} I^{2}+G_{0} \beta I+G_{1}},
\end{aligned}
$$


where

$$
G_{0}=(\xi \omega+\mu \omega+\mu+\theta)
$$

and

$$
G_{1}=(\xi+\mu+\theta) \mu
$$

The fourth and final equations in (20) lead to

$$
\begin{gathered}
E^{*}=\frac{(\tau+\mu+\delta) I}{p \beta I+\kappa}, \\
R^{*}=\frac{\tau I}{\sigma \beta I+\mu} .
\end{gathered}
$$

Substituting $S^{*}, V^{*}, E^{*}$, and $R^{*}$ into the third equation of (20), the endemic equilibrium condition becomes a cubic polynomial equation of $I^{*}$, satisfying the equation below:

$$
f(I)=m_{1} I^{3}+m_{2} I^{2}+m_{3} I+m_{4}=0,
$$

where

$$
\begin{aligned}
& m_{1}=\beta^{3} p \sigma(d+\mu) I^{3} \\
& m_{2}=\left(((p+1) \sigma+p) \beta^{2} \mu^{2}+(((p+1) d+p \xi+\kappa+\delta) \sigma+p(\delta+\tau)) \beta^{2} \mu\right. \\
& +(\kappa \delta \beta+\beta p(-\Lambda \beta \omega+\delta \xi)) \beta \sigma) I^{2} \\
& m_{3}=\left((\sigma+p+1) \beta \mu^{3}+((\delta+k+\tau+\xi) \sigma+(p+1) \delta+\kappa+(\delta+\xi) p+\tau) \beta \mu^{2}+\right. \\
& \left.\left(-\omega \Lambda p \beta^{2}+(((\kappa+\xi) \delta+\xi(\kappa+\tau)) \sigma+(p \xi+\kappa)(\delta+\tau)) \beta\right) \mu+\kappa(-\Lambda \beta \omega+\delta \xi) \beta \sigma\right) I \\
& m_{4}=\mu(\xi+\mu+\theta)(\mu+\kappa)(\mu+\tau+\delta)(1-R v) .
\end{aligned}
$$

Here, $R v$ is the effective basic reproduction number given in (15).

It is clear that I is given by the positive real roots of the polynomial (25). The number of possible positive real roots of the cubic polynomial (25) depends on the signs of $m_{2}, m_{1}$, and $m_{0}$. This will be investigated by employing Descarte's rule of sign. A number of possibilities are illustrated in Table 1 . Therefore, the outcomes can be established by the following theorem.

Table 1. Number of possible positive roots of the cubic polynomial Equation (25) .

\begin{tabular}{cccccccc}
\hline Cases & $m_{\mathbf{3}}$ & $m_{\mathbf{2}}$ & $m_{\mathbf{1}}$ & $m_{\mathbf{0}}$ & $\boldsymbol{R}_{v}$ & Changes in Sign & Total Possible Positive Roots \\
\hline 1 & + & - & - & - & $R v>1$ & 1 & 1 \\
2 & + & + & - & - & $R v>1$ & 1 & 1 \\
3 & + & + & + & - & $R v>1$ & 1 & 1 \\
4 & + & - & + & - & $R v>1$ & 3 & 1,3 \\
5 & + & - & - & + & $R v<1$ & 2 & 0,2 \\
6 & + & + & - & + & $R v<1$ & 2 & 0,2 \\
7 & + & - & + & + & $R v<1$ & 2 & 0,2 \\
8 & + & + & + & + & $R v<1$ & 0 & 0 \\
\hline
\end{tabular}

Theorem 4. The TB model system (1) has:

1. A unique endemic equilibrium when $R v>1$ and cases $1-3$ are satisfied;

2. One or more than one endemic equilibrium when $R v>1$ and cases 5-7 are satisfied; and

3. No endemic equilibrium when $R v<1$ and case 8 shows that all the coefficients are positive.

\subsection{Threshold Analysis and Vaccine Impact}

Given that we considered the TB vaccine to be imperfect, however, it is instructive to choose whether or not it wide-spread use in a population is consistently assured or not. To assess the impact of such a vaccine on the transmission of the disease, a qualitative approach can be adopted, by differentiating the expression $R v$ with respect to the fraction of the individuals vaccinated at the steady state and introducing 


$$
\eta=\frac{V^{*}}{N^{*}} .
$$

We can consider $R v$ as a function of $\eta$; that is,

$$
R v=R v(\eta)=R_{0}\left(\frac{\eta+(1-\omega) \xi}{\xi+\mu}\right)=R_{0}(1-\eta \omega) .
$$

Observing that $R v \leq R_{0}$, with equivalence only if $\xi=0$ (i.e., $\eta=0$ ) or $\omega=0$. That is to say, even though the vaccine is imperfect, it is notable that a decrease in reproduction number implies a decrease in the disease epidemic. With $\xi>0$ and $\omega>0$, a reduction in disease burden would be observed.

As $R v \leq 1$ is a essential and proper condition for the elimination of diseases (see Section 4.2 and Theorem 3), accordingly, the following condition on $\eta$ is equally fundamental and sufficient for control:

$$
\eta \geq \frac{1}{\omega}\left(1-\frac{1}{R_{0}}\right)=\eta_{c}
$$

Section 4.2 and Theorem 3 can be joined to give the following outcome:

Lemma 1. TB is removable from the population if $\eta>\eta_{c}$.

Our concept for the fraction vaccinated at equilibrium point, $\eta_{c}$, is the same as that derived in $[60,72]$. The critical value, $\eta_{c}$, is graphically illustrated as a function of $\omega$, for several $R v$ values, in Figure 2. Equation (26) indicates that both the vaccinated fraction, $\eta$, and the vaccine efficacy, $\omega$, assume key roles in the reduction of $R v$, and both must be high in order to diminish the value of $R v$ to less than one and, thus, manage the disease. Inequality (27) can also be reconfirmed as

$$
\eta \omega \geq 1-\frac{1}{R_{0}}
$$

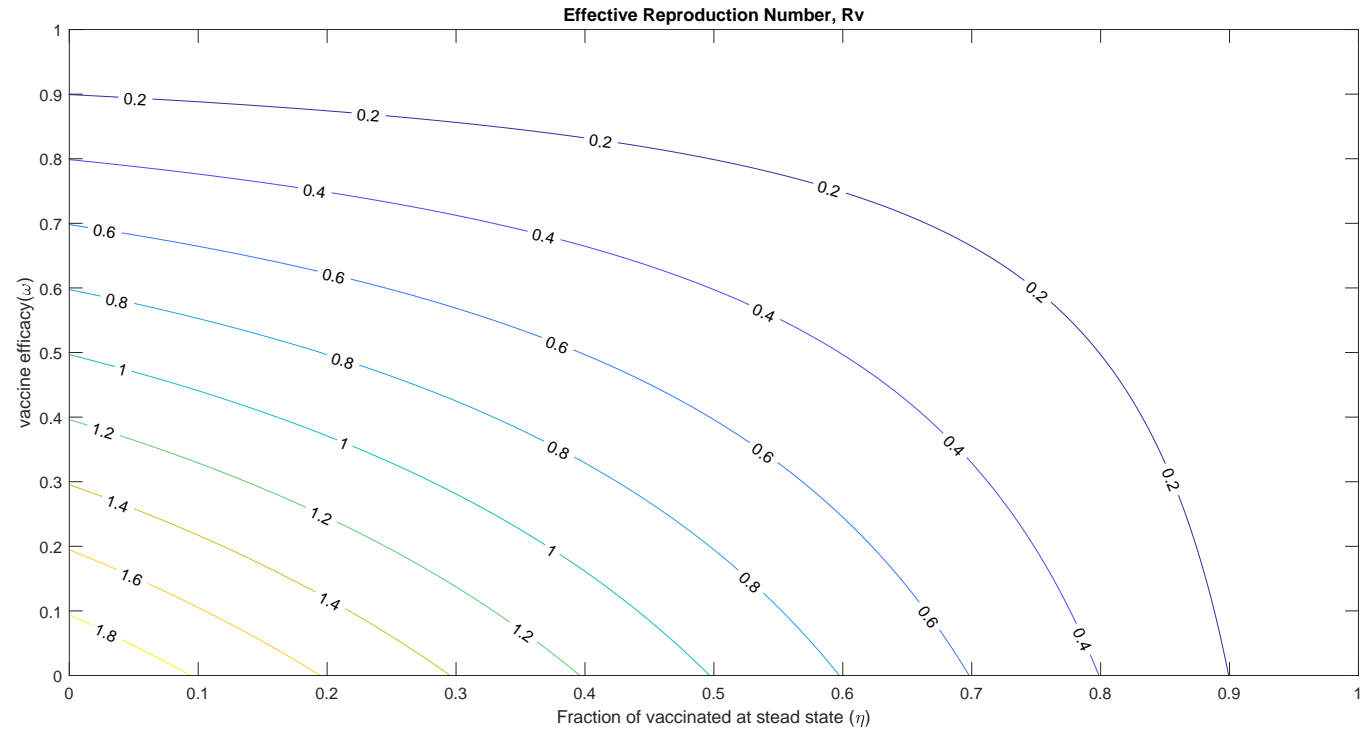

Figure 2. Simulation of the system model (1) showing a contour plot of $R v$ as a function of vaccinated individuals at steady-state $\left(\eta=\frac{V^{*}}{N^{*}}\right)$ and vaccine efficacy $\omega$. Parameter values used are as given in Table 2.

A contour plot of the effective threshold $R v$, as a function of vaccine efficacy $\omega$ and the fraction of individuals vaccinated at a steady-state $\left(\eta=\frac{V^{*}}{N^{*}}\right)$, is illustrated in Figure 2. For 
the set of the parameters used in these simulations, the contours show a marked decrease in effective basic reproduction number $(R v)$ with increasing vaccine efficacy $(\omega)$ and fraction of individuals vaccinated $(\eta)$. Significantly, high efficacy and vaccine coverage are needed to effectively control the disease in the population (i.e., to achieve $R v<1$ ).

In terms of the vaccination rate, $\xi$, the inequality (27) can be used. This is done by taking note, as a matter of first importance, that the center representation of Equation (26) may be a diminishing function of $\xi$; thus, it is limited by letting $\xi$ have no bound. Adopting the limit as $\xi$ approaches infinity, we see that this expression is consistently more noteworthy than $(1-\omega) R_{0}$. Subsequently, if $(1-\omega) R_{0} \geq 1$, at that point, no measure of vaccination can force $R v$ to less than one. Similarly, if $(1-\omega) R_{0}<1$, at that point, the condition

$$
\xi \geq \frac{\mu\left(R_{0}-1\right)}{1-(1-\omega) R v}=\xi_{o}
$$

gives $R v \leq 1$. Obviously, this condition assumes $R_{0}>1$. Disease control without vaccination follows if $R_{0} \leq 1$ (by Section 4.2 and Theorem 3 and the proof that $R v \leq R_{0}$ ). By (28), it is easy to see that $R v \leq 1$ if $\xi \geq \xi_{0}$, and $R v>1$ if $\xi<\xi_{0}$. Therefore, we obtained the following results:

Lemma 2. If $(1-\omega) R_{0}<1$ and $\xi \geq \xi_{0}$, then $T B$ will eradicated from the population. If $(1-\omega) R_{0} \geq 1$, at that point, no measure of vaccination will prevent a TB epidemic in the population.

\subsection{Global Stability of DFE, $P_{0}$, for $R v \leq 1$}

Motivated by Ullah et al. [3], we construct a Lyapunov function to prove the global stability of the DFE. We define the following:

$$
L=\kappa E+(\kappa+\mu) I .
$$

Differentiating $L$ with respect to time and observing that $S+\omega V<1$, we obtain

$$
\begin{gathered}
\frac{d L}{d t}=\kappa \frac{d E}{d t}+(\kappa+\mu) \frac{d I}{d t} \\
=\beta \kappa[S+\omega V] I-(\kappa+\mu)(\mu+\tau+\delta) I \\
=(\kappa+\mu)(\mu+\tau+\delta)(R v-1) I .
\end{gathered}
$$

Therefore, if $R v<1$, then $\frac{d L}{d t}$ is non-positive. Hence, the largest compact invariant set in $\Omega$ is the singleton set $P_{0}$. Therefore, LaSalle's invariance principle [73] implies that $P_{0}$ is globally asymptotically stable in $\Omega$.

The above explanation shows that TB infection can be eliminated in a population if and only if $R v<1$.

\subsection{Analysis of Backward Bifurcation}

In the model system (1), the existence of multiple TB persistence equilibria $P^{*}$ for $R v$ suggests the possibility of the backward bifurcation phenomenon. Epidemiologically, it suggests that $R v$ is not sufficient to decide whether or not TB would persist; instead, it depends on the initial population size of the individuals when $R v<1$. Our goal is to investigate and set a threshold for the presence of backward bifurcation in system (1). To do this, we utilize the notable results of Castillo-Chavez and Song [12]. In a simpler way, we rewrite our system (1) by choosing $S=x_{1}, V=x_{2}, E=x_{3}, I=x_{4}, R=x_{5}$, where we set $X=\left(x_{1}, x_{2}, x_{3}, x_{4}, x_{5}\right)^{T}$ and $F=\left(f_{1}, f_{2}, f_{3}, f_{4}, f_{5}\right)^{T}$. 


$$
F(X)=\left(\begin{array}{c}
\Lambda+\theta x_{2}-\xi x_{1}-\beta x_{1} x_{4}-\mu x_{1} \\
\xi x_{1}-\omega \beta x_{2} x_{4}-\theta x_{2}-\mu x_{2} \\
\beta x_{1} x_{4}+\omega \beta x_{2} x_{4}-p \beta x_{5} x_{4}-(\kappa+\mu+\alpha) x_{3}+\sigma \beta x_{4} x_{3} \\
p \beta x_{3} x_{4}+\kappa x_{3}-(\mu+\tau+\delta) x_{4} \\
\tau x_{4}-\sigma \beta x_{5} x_{4}-\mu x_{5}
\end{array}\right)=\left(\begin{array}{c}
f_{1} \\
f_{2} \\
f_{3} \\
f_{4} \\
f_{5}
\end{array}\right)
$$

The Jacobian matrix of the system at disease-free equilibrium $P_{0}=\left(\frac{(\mu+\theta) \Lambda}{\mu(\xi+\mu+\theta)}\right.$, $\left.\frac{\Lambda(\xi)}{\mu(\xi+\mu+\theta)}, 0,0,0\right)$, is

$$
J_{P_{0}}=\left(\begin{array}{ccccc}
-(\mu+\xi) & \theta & 0 & -\frac{\beta(\mu+\theta) \Lambda}{\mu(\xi+\theta+\mu)} & 0 \\
\xi & -(\mu+\theta) & 0 & -\frac{\omega \beta \xi \Lambda}{\mu(\xi+\theta+\mu)} & 0 \\
0 & 0 & -(\kappa+\mu) & \frac{\omega \beta \xi \Lambda}{\mu(\xi+\theta+\mu)}+\frac{\beta(\mu+\theta) \Lambda}{\mu(\xi+\theta+\mu)} & 0 \\
0 & 0 & \kappa & -(\mu+\tau+\delta) & 0 \\
0 & 0 & 0 & \tau & -\mu
\end{array}\right) .
$$

We chose $\beta$ as a bifurcation parameter when $R_{0}=1$, giving $\beta^{*}=\frac{\mu(\xi+\mu+\theta)(\mu+\kappa)(\mu+\tau+\delta)}{\Lambda(\xi \omega+\mu+\theta}$. At the threshold value, the changed-over matrix $J_{P_{0}}$ has one simple zero eigenvalue, whose right and left eigenvectors are given by $\mathbf{w}=\left(\left[\frac{\omega \beta \Lambda}{\mu(\xi+\mu+\theta)}-\frac{(\mu+\theta) \beta \Lambda\left(A_{0}(\delta+\mu+\theta)\right)}{\mu^{2}(\xi+\mu+\theta)^{2}(\delta+\mu+\theta)}\right] w_{4}\right.$, $\left.\left[\frac{-\beta \Lambda \xi\left(A_{0}(\delta+\mu+\theta)\right)}{\mu^{2}(\xi+\mu+\theta)^{2}(\delta+\mu+\theta)}\right] w_{4},\left[\frac{\beta \Lambda(\omega \xi+\mu+\theta)-\mu(\xi+\mu+\theta)(\delta+\mu+\theta)}{\mu^{2}(\xi+\mu+\theta)}\right] w_{4}, w_{4},\left[\frac{\tau}{\mu}\right] w_{4}\right)$ and $\mathbf{v}=\left(0,0,\left[\frac{\kappa}{(\kappa+\mu)}\right] v_{4}, v_{4},\left[\frac{(\kappa+\mu)(\delta+\mu+\tau)-B_{0} \kappa}{\tau(\kappa+\mu)}\right]\right)$, respectively,

Where

$$
\begin{gathered}
A_{0}=(\mu+\delta)(\xi+\mu+\theta) \omega+(\mu+\theta), \\
B_{0}=\frac{\beta \Lambda(\omega \xi+\theta+\mu)}{\mu(\xi+\theta+\mu)} .
\end{gathered}
$$

In order to obtain the following quantity stated by Castillor-Chavez and Song in their Theorem 4.1, we have

$$
\boldsymbol{a}=\sum_{k, i, j=1}^{5} v_{k} w_{i} w_{j} \frac{\partial^{2} f_{k}}{\partial x_{i} \partial x_{j}}(0,0)
$$

and

$$
\boldsymbol{b}=\sum_{k, i=1}^{5} v_{k} w_{i} \frac{\partial^{2} f_{k}}{\partial x_{i} \partial \beta}
$$

which are determined to be

$$
\boldsymbol{a}=2 \beta w_{4}\left(\omega v_{2} w_{2}+\omega w_{3} w_{2}-p v_{3} w_{3}+p v_{4} w_{3}+\sigma v_{3} w_{5}-v_{1} w_{1}+v_{3} w_{1}\right)
$$

and

$$
\boldsymbol{b}=v_{3} w_{4}\left(\frac{\omega \xi \Lambda}{\mu(\xi+\theta+\mu)}+\frac{(\mu+\theta) \Lambda}{\mu(\xi+\theta+\mu)}\right)>0 .
$$


As indicated by the result shown in [12], our system experiences backward bifurcation at $\beta=\beta^{*}$, only when both $a$ and $b$ are positive at $\left(P_{0}, \beta^{*}\right)$. Obviously, $b$ is consistently positive. Hence, the positivity of $a$ offers the threshold circumstance for the phenomenon of backward bifurcation.

\section{Numerical Simulation}

Our motivation in this study was to determine how the rate of vaccination, exogenous re-infection, and transmission rate influence the model framework. Some of the numerical simulation findings are presented in this section basically to illustrate the numerical results obtained. The model parameter values chosen for the simulation are given in Table 2.

Table 2. Parameter and Values used for model (1).

\begin{tabular}{|c|c|c|c|c|}
\hline Parameters & Descriptions & Values & B & Unit \\
\hline$\Lambda$ & Recruitment of individual either by immigration or birth & 5 & [22] & year $^{-1}$ \\
\hline$\omega$ & Reduction in risk of infection due to vaccination & $0-1,0.2,0.90$ & {$[74,75]$} & year $^{-1}$ \\
\hline$\theta$ & The rate at which vaccine wanes & $0.067,0.1$ & {$[76-79]$} & year $^{-1}$ \\
\hline$\xi$ & The rate at which susceptible individuals are vaccinated & $0,0.1,0.98,0.95$ & {$[39,40,80]$} & year $^{-1}$ \\
\hline$\mu$ & Natural death rate & 0.15 & [21] & - \\
\hline$\delta$ & Disease-induced death rate due to $\mathrm{TB}$ & 0.12 & {$[18,81]$} & year $^{-1}$ \\
\hline$\beta$ & Transmission rate & variable & - & - \\
\hline$\sigma$ & Reinfection among the treated individuals & $0-1$ & {$[34,82,83]$} & - \\
\hline$\kappa$ & Progression rate & 0.02 & [21] & year $^{-1}$ \\
\hline$\tau$ & Recovery rate & $1.5-3.5$ & [35] & year $^{-1}$, day $^{-1}$ \\
\hline$p$ & Exogenous re-infection & $0-1$ & {$[17,23,34,83]$} & - \\
\hline
\end{tabular}

The parameter values presented are biologically feasible and the vast majority of them were taken from important literature relating to TB transmission models. Numerical simulation and bifurcation analyses were performed using MATLAB. The sensitive quantity value (i.e., the effective basic reproduction number $R v$ ) was determined by using the set of parameter values stated in Table 2; with $\beta=0.8$ and $\xi=0.3$ we obtained $R v \approx 0.8708<1$, and found that this system posses both the disease-free equilibrium point $P_{0}=(26,7,0,0,0)$ and two endemic equilibrium points, respectively at $P_{01}(16.5384,15.2210,9.7436,0.1483$, $15.3825)$ and $P_{02}(2.8433,0.2239,34.0369,3.3738,7.9885)$. The corresponding eigenvalues of the Jacobian matrix $J_{P 0}$ were $-0.1500,-0.05125,-0.1510,-0.4500,-3.3887$, which are all negative and, thus, show that the disease-free equilibrium point $P_{0}$ is locally asymptotically stable; see Figure 3. Thus, the eigenvalues that lead to two endemic equilibria for $P_{1}^{*}$ are $2.051214029+0.878706126 \mathrm{i},-0.5511435244 \times 10^{-1}+0.1715247095 \mathrm{i},-0.1522994290$ $+0.5977159283 \mathrm{i},-0.1993628469-0.0 .6497115331 \mathrm{i}$, and $0.3730450707 \mathrm{i}-0.8057137074 \mathrm{i}$, respectively. The previous set of eigenvalues all had negative real parts, while the other set had positive values. The first endemic equilibrium was locally stable and the other was unstable, as a result. This confirms the presence of a persistent TB stable steadystate, despite $R v<1$; thus, the convergence of the trajectory depends on the population's initial size. To demonstrate this graphically, the solution trajectories determined using two different initial conditions are represented in Figure 4, with two different initial population sizes. 


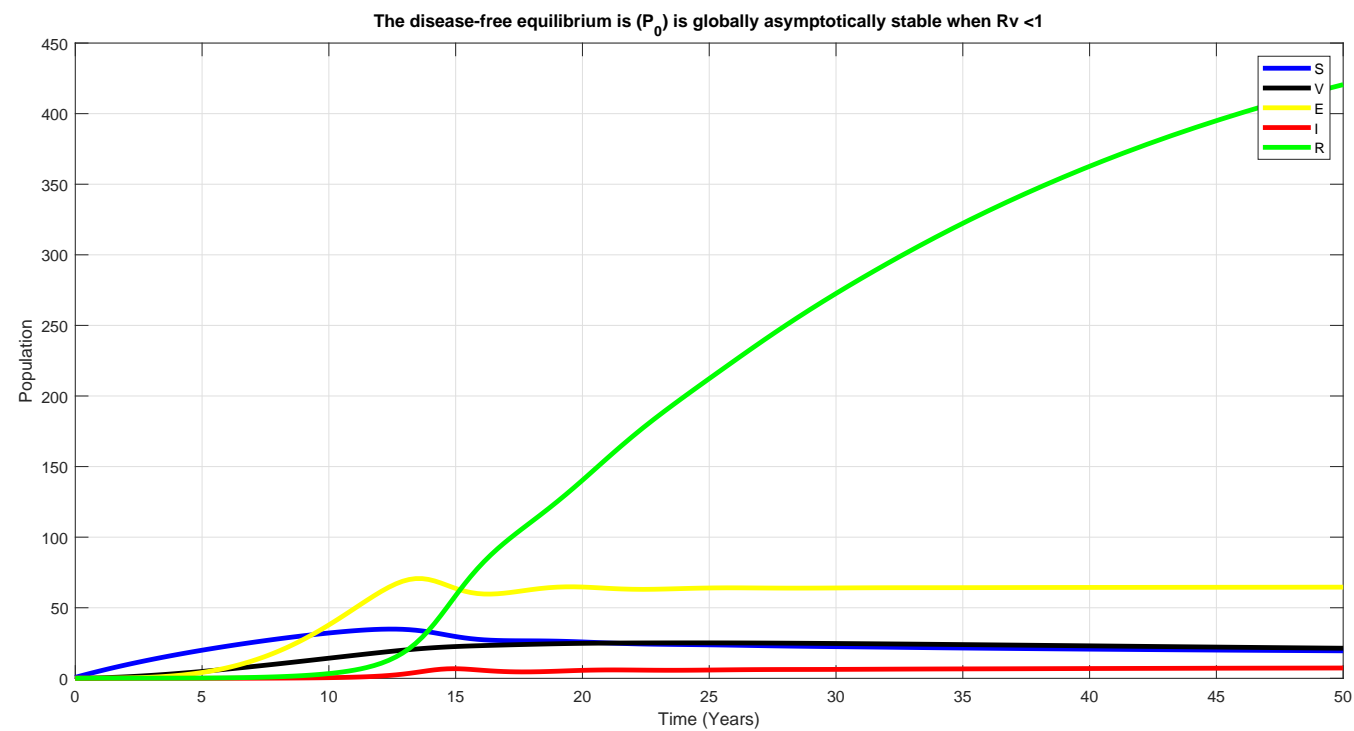

Figure 3. Solution trajectories with $\xi=0.3$ and $\beta=0.8$ with other parameters fixed: $\mu=0.15$, $\Lambda=5, p=0.17, \beta=1, \kappa=0.02, \delta=0.12, \sigma=0.7, \tau=3, \omega=0.8$, and $\theta=0.2$, which gives $R v=0.6052<1$.
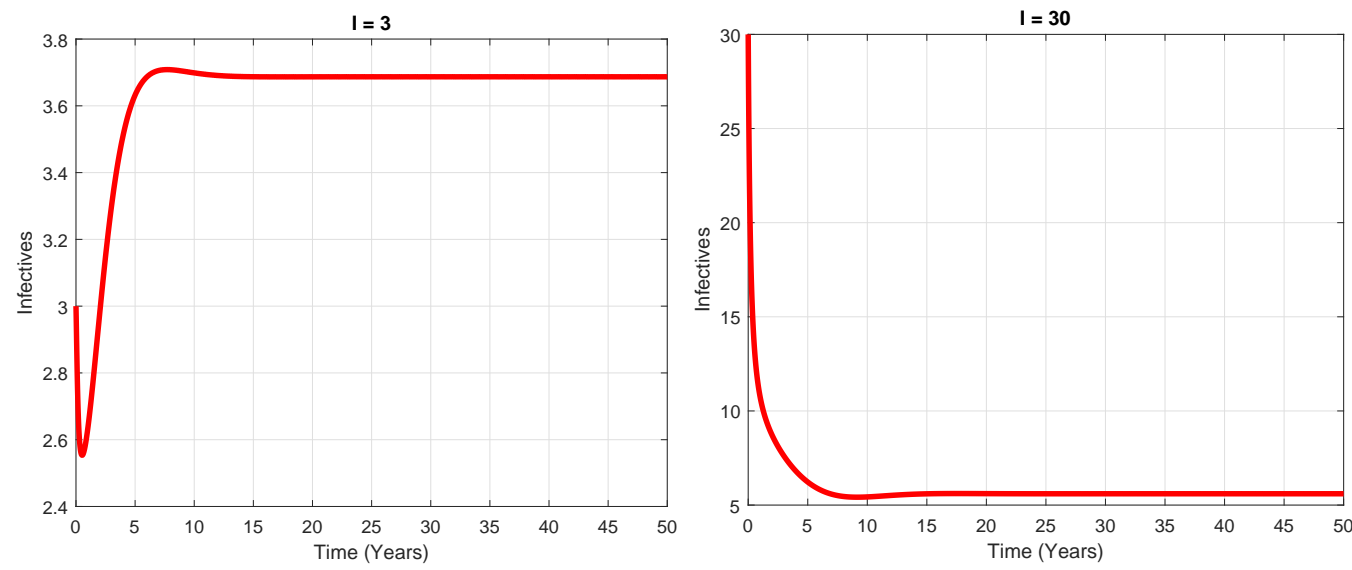

Figure 4. Time-series plot for infectious compartment $(I)$ for $\beta=1.05$ and $\xi=0.1$, with all parameter values listed in Table 2 . The left curve converges to the endemic equilibrium $P^{*}$ whereas right curve converges to the disease-free equilibrium point $P_{0}$ with different initial sizes of the population.

\section{Discussion}

Figure 5 represents the variation of the system model (1) as a function of time (years) for $\beta=1.05$ and $\xi=0.1$, which gives $R v=1.0355>1$. The figure shows that the global stability of the endemic equilibrium $P^{*}$ is locally asymptotically stable. From the diagram, it can be seen that the trajectories of the solution of the system model (1) converge to the endemic equilibrium point $P^{*}$; this shows that TB transmission persists in the population if $R v>1$, which verifies the justification for the statement that endemic equilibrium is GAS if $R v>1$.

Figure 3 illustrates the variation of the system model (1) as a function of time (years) for $\beta=0.8$ and $\xi=0.3$, which gives $R v=0.6052<1$. This figure depicts the local stability of the TB-free equilibrium. The diagram shows that the trajectories of the solution of the system model (1) converge to the disease-free equilibrium $P_{0}$. This figure implies that the disease will not invade the population if $R v<1$, verifying that the disease-free equilibrium is GAS if $R v<1$. 


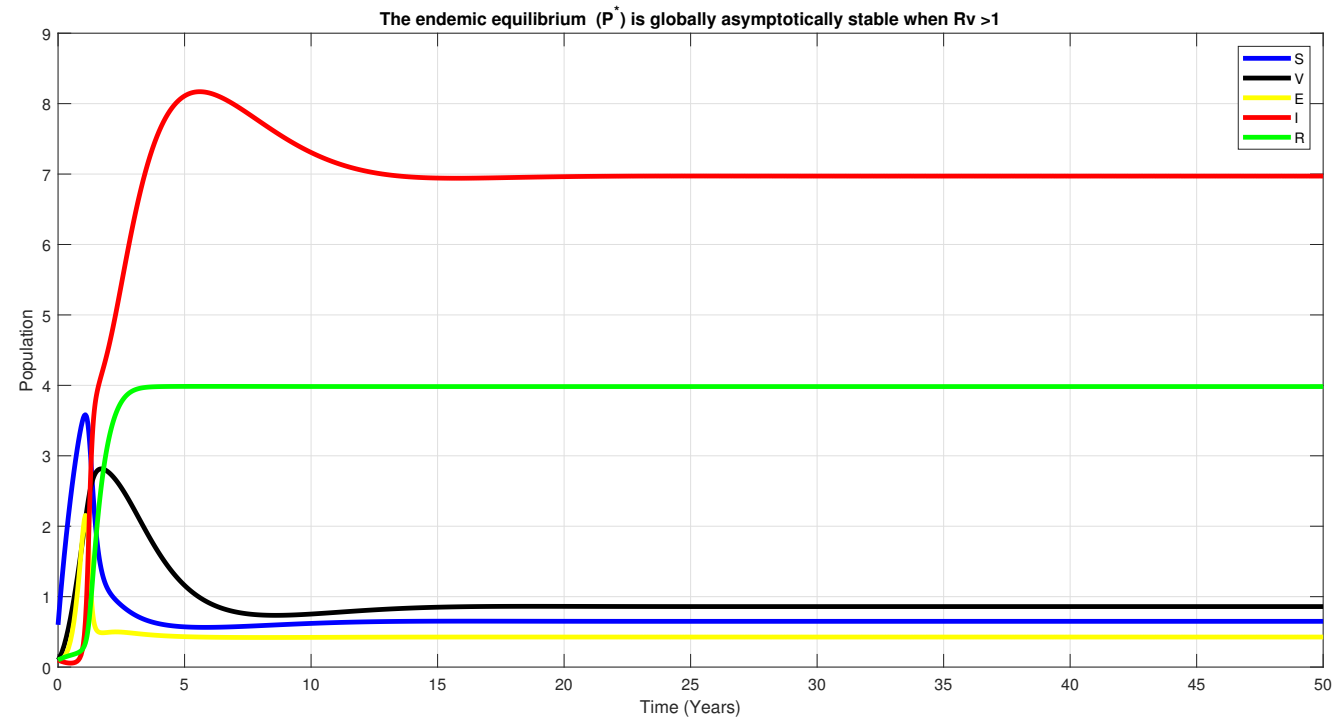

Figure 5. Solution trajectories with $\xi=0.1$ and $\beta=1.05$ with other parameters fixed: $\mu=0.15, \Lambda=5$, $p=0.17, \kappa=0.02, \delta=0.12, \sigma=0.7, \tau=3, \omega=0.8$, and $\theta=0.2$, which gives $R v=1.0355>1$.

Figure 4 shows the time-series plot for the infected population (I) for $\beta=1.05$ and $\xi=0.1$, with all other parameter values as specified in Table 2 . The left curve converges to the endemic equilibrium $P^{*}$, whereas the right curve converges to the disease-free equilibrium point $P_{0}$ with different initial population sizes.

To examine the backward bifurcation phenomenon numerically, we first figured out that both $a$ and $b$ must be non-negative (as stated in Section 5.3); otherwise, the system (1) undergoes backward bifurcation. Figure 6 illustrates the backward bifurcation diagram of the system (1) as the effective basic reproduction number $(R v)$ varies against the infected population (I). The first (top-left) diagram of Figure 6 depicts the bifurcation diagram at $\beta=1.05$. In general, there are several branches of steady-states: (i) the upper branch corresponds to stable endemic equilibria (EEP); (ii) the middle branch represents unstable EEP; and (iii) the lower branch corresponds to disease-free equilibria, DFE, which can be stable or unstable, depending on the magnitude of $(R v)$. When $R v>1$, only EEP is stable and this situation leads to an outbreak of TB. It is also clear that, when $R v<1$, DFE is stable in this case; consequently, this situation leads to elimination of the disease. To better understand the transmission dynamics of system (1), we plot some time-series diagrams in Figure 7 (left) with the rate of transmission $\beta$ varying. One of the possibilities when the magnitudes of $\beta$ are high is that this situation would lead to the persistence of TB (i.e., the trajectories converge to EEP) in this epidemiological system. However, as the transmission rate $\beta$ decreases, we observed that the backward bifurcation loses. This results in small $R v$ quantities, as shown by the bottom-left (respectively, bottom-right) of Figure 6 with $\beta=0.85$ (respectively, $\beta=0.8$ ) and $R v=0.9466$ (respectively, $R v=0.8909$ ); consequently, the TB disease is eradicated and the DFE is stable in the long-term.

From Figure 8, it can be seen that, when the effective reproduction number $R v$ is between 0 and $R v c$, the DFE is stable; while, for $R v c<R v<1$, there exists bi-stability, where either the DFE is stable or the EEP is stable. Rvc is a threshold quantity where the two non-trivial endemic equilibria collide and annihilate each other, leaving the DFE as the only stationary solution. However, in order to observe the impact of vaccination coverage $\xi$ clearly, the bifurcation diagram was drawn for various $\xi$ values. Hence, Figure 8 gives a good description of the bifurcation diagram, revealing that the vaccination coverage $\xi$ plays a significant role in the dynamics of TB transmission. It can be seen that, when the vaccination coverage was increased, the bifurcation diagram steadily shifted towards the right, causing the $R v$ value to increase. This implies that, if the $R v c$ value is sufficiently large, then backward bifurcation will not exist. Hence, in this situation, $R v$ being less than unity is sufficient to eradicate the community disease. However, when the likelihood 
of vaccination increases, this normally brings about a decrease in effective reproduction number, which causes a number of a new infections; the critical detection level corresponds to 1 .
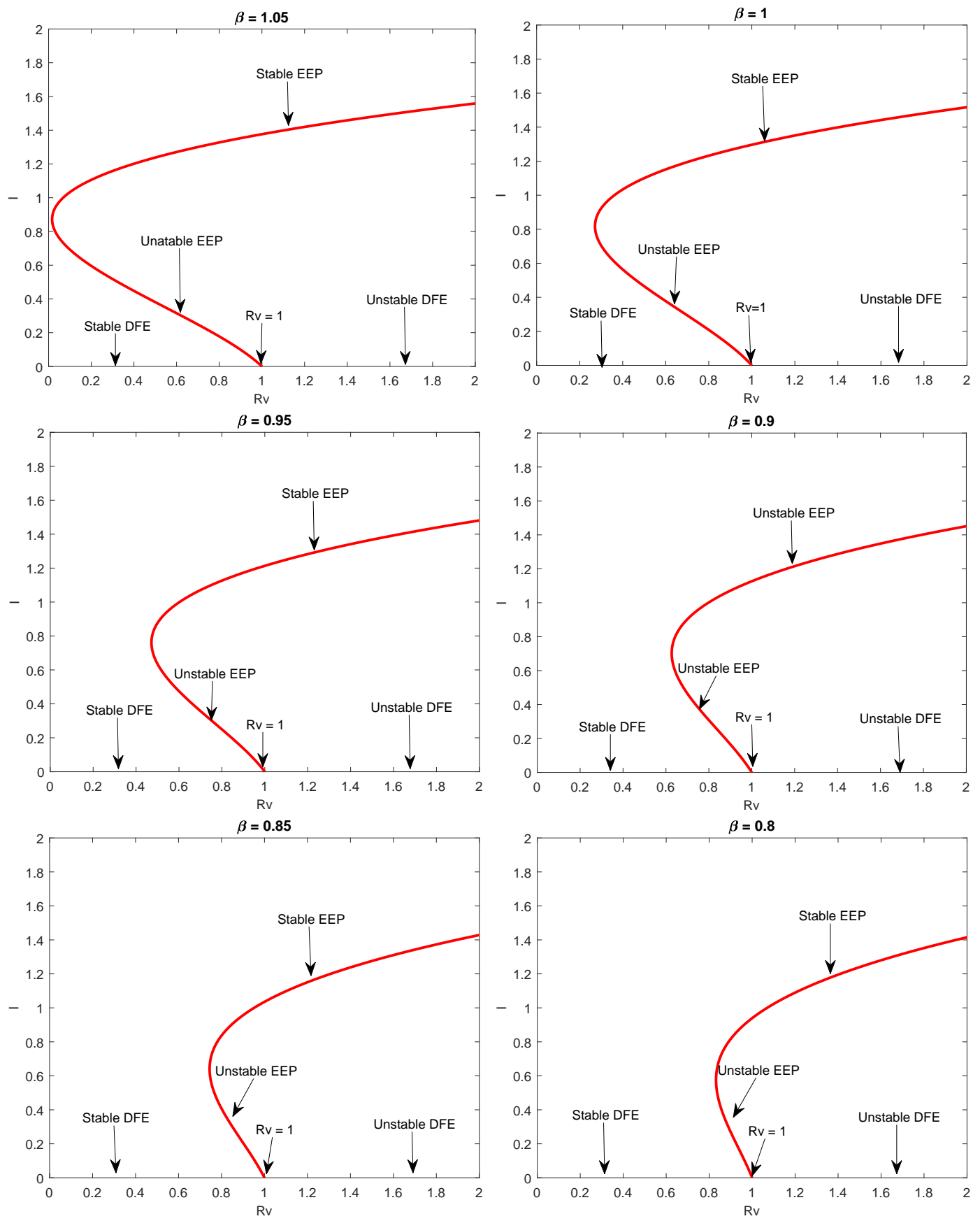

Figure 6. Backward Bifurcation diagram of system model (1), showing different types of behaviour. As the bifurcation parameter $\beta$ changes from the top left, different values of $\beta$ are used in clockwise direction, respectively: $\beta=1.05, \beta=1$, $\beta=0.95, \beta=0.9, \beta=0.85$, and $\beta=0.8$, while the other parameters values are fixed: $\mu=0.15, \Lambda=5, p=0.17, \kappa=0.02$, $\sigma=0.7, \tau=3, \omega=0.2, \delta=0.12 \xi=0.1$, and $\theta=0.2$. The symbols EEP and DFE represent the endemic equilibrium point and disease-free equilibrium point, respectively.

Figure 2 demonstrates the simulation of the system model (1), showing a contour plot of $R v$ as a function of vaccinated individuals at steady-state $\left(\eta=\frac{V^{*}}{N^{*}}\right)$ and vaccine efficacy $\omega$. The parameter values used are given in Table 1 . The contours show a marked decrease in effective basic reproduction number $(R v)$ with increasing vaccine efficacy $(\omega)$ 
and fraction of individuals vaccinated $(\eta)$. If at least $50 \%$ of the population vaccinated at steady-state $\eta$ with about $50 \%$ vaccine efficacy, the effective reproduction number of the disease can be brought below 1 , which demonstrates that the infection will ultimately cease to exist in the population. Notably, high efficacy and vaccine coverage are required for the effective control of the disease in the population (i.e., to achieve $R v<1$ ).
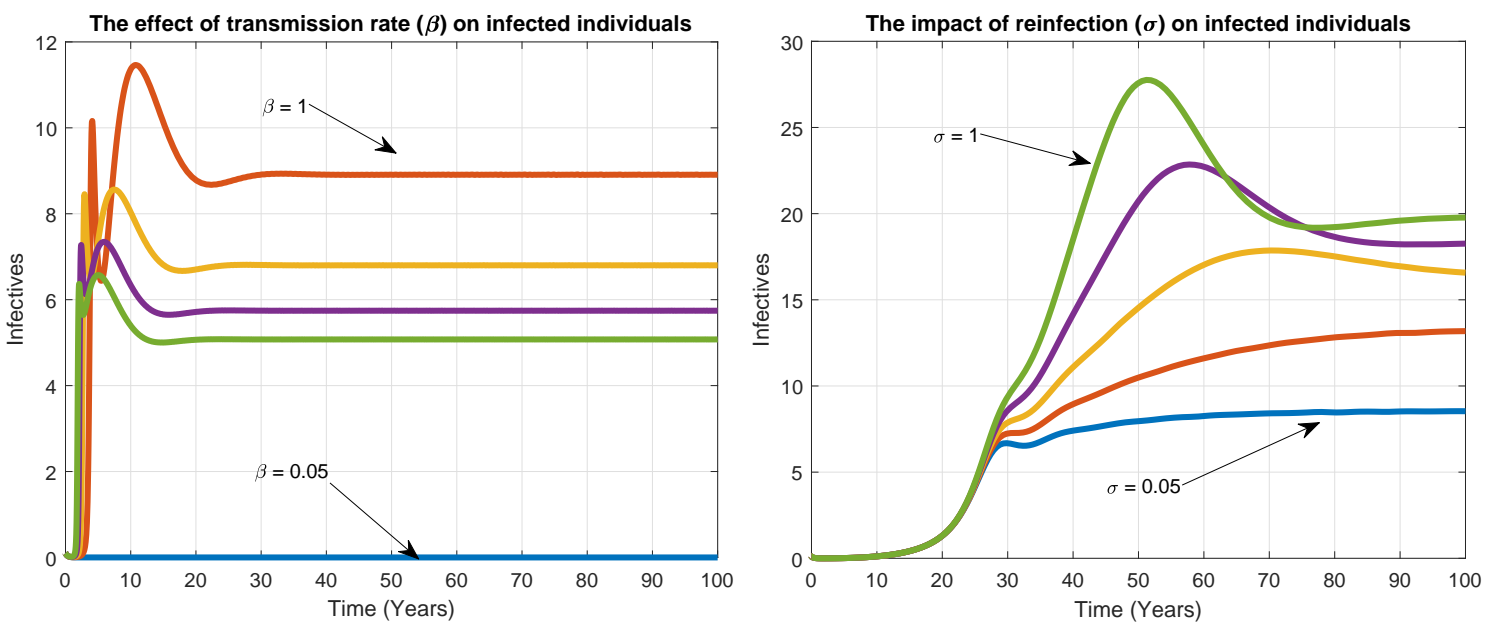

Figure 7. Impact of transmission rate $\beta$ and re-infection $\sigma$ among treated individuals on infected population using the same parameters as in Table 2, while $\beta$ and $\sigma$ are varied.

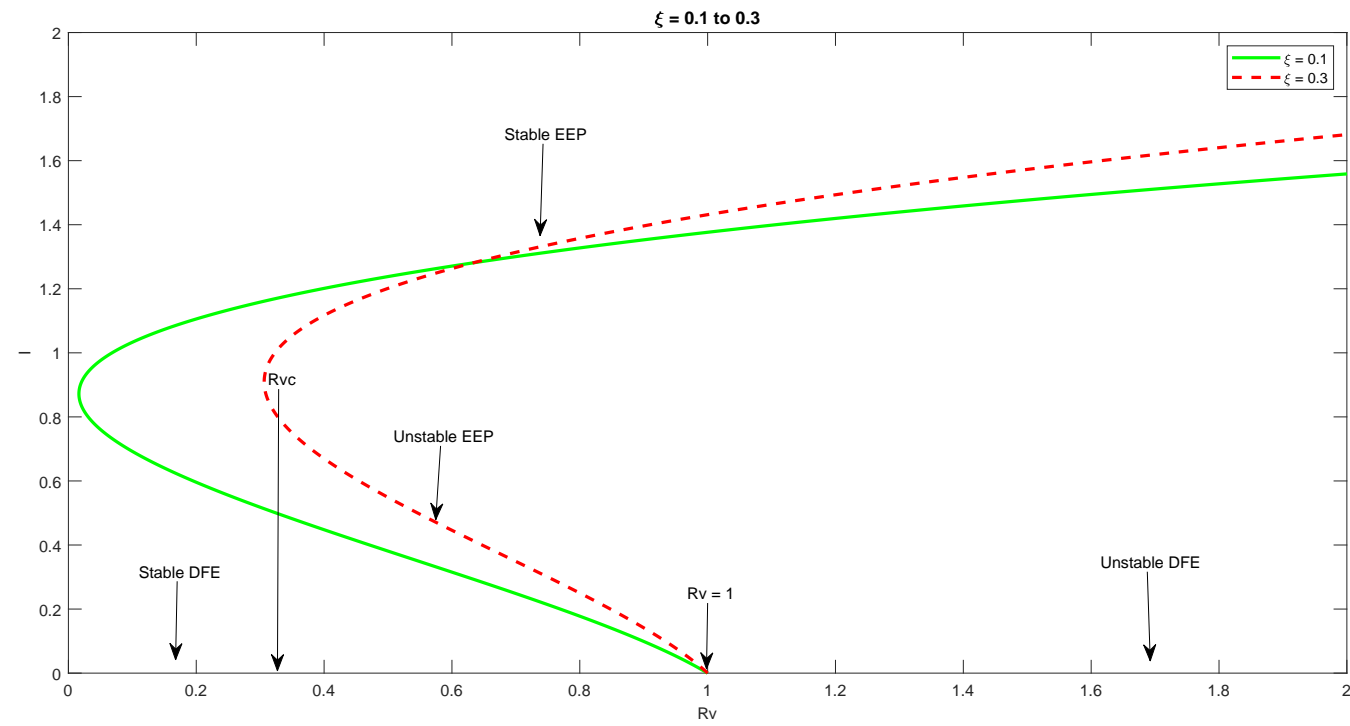

Figure 8. Backward Bifurcation diagram showing effective basic reproduction number against infected individuals varying with vaccination coverage, with $\xi=0.1$ and $\xi=0.3$ with other parameters fixed: $\mu=0.15, \Lambda=5, p=0.17, \beta=1 \kappa=0.02, \delta=0.12, \sigma=0.7, \tau=3, \omega=0.8$, and $\theta=0.2$. The symbols EEP and DFE represent the endemic equilibrium point and disease-free equilibrium point, respectively.

The effects of vaccination coverage $\xi$, exogenous re-infection $(p)$, and transmission rate $(\beta)$ and re-infection among the treated people $(\sigma)$ are shown in Figures 7, 9 and 10, respectively. In Figure 9, we study the impact of vaccination coverage $\xi$ on infected and exposed individuals, with $\xi$ ranging from 0.1 to 0.9. From both diagrams in Figure 9, the highest value of $\xi$ significantly reduced the spread of infection in a population, but did not completely lead to the elimination of TB infections. Figure 10 shows the effects of exogenous re-infection; that is, acquiring a new infection from another infectious individual. We noticed that the highest value of $p=1$ showed that the infection converged to endemic state. We further studied the effects of transmission rate $\beta$ and re-infection among the 
treated individuals $\sigma$. It can be clearly observed that, with a lower threshold value of the transmission rate $\beta$ and re-infection among the treated individuals $\sigma$, the infection rate is low. For high values of $\beta$ and $\sigma$, the infection rate is higher; see Figure 10, which shows that the viability of $\beta$ and $\sigma$ is helpful for the control of TB infection.

Figure 11 (left) shows the impact of varying reductions in susceptibility to disease by the vaccinated individuals $\omega$ in the proportion of the infected class, ranging between 0 to 1. Of course, increasing the value of $\omega$ from 0 to 1 significantly reduced the proportion of infected individuals. In addition, Figure 11 (right) shows a more terrible situation, whereby an increase in the rate of vaccine wane $\theta$ led to an increase in the proportion of infected individuals.

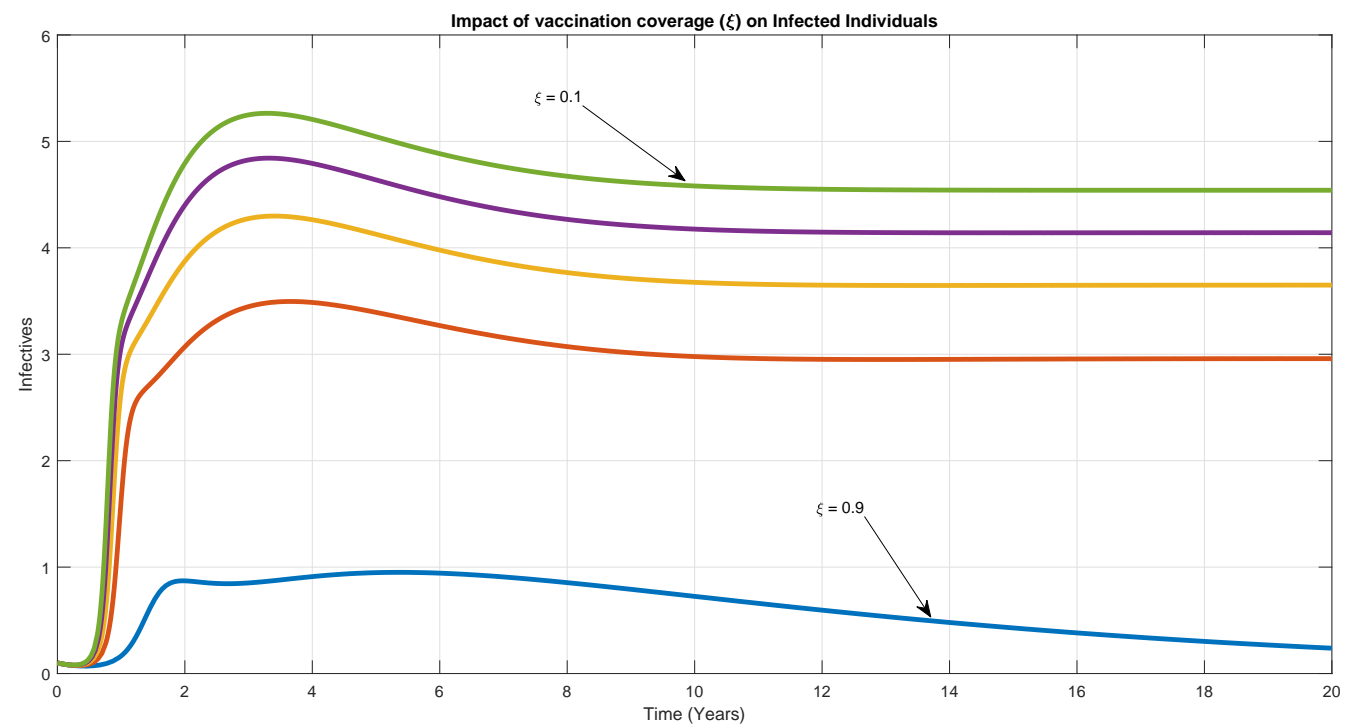

Figure 9. Impact of vaccination coverage on infected individuals using the same parameters as in Table 2 while $(\xi)$ is varied.

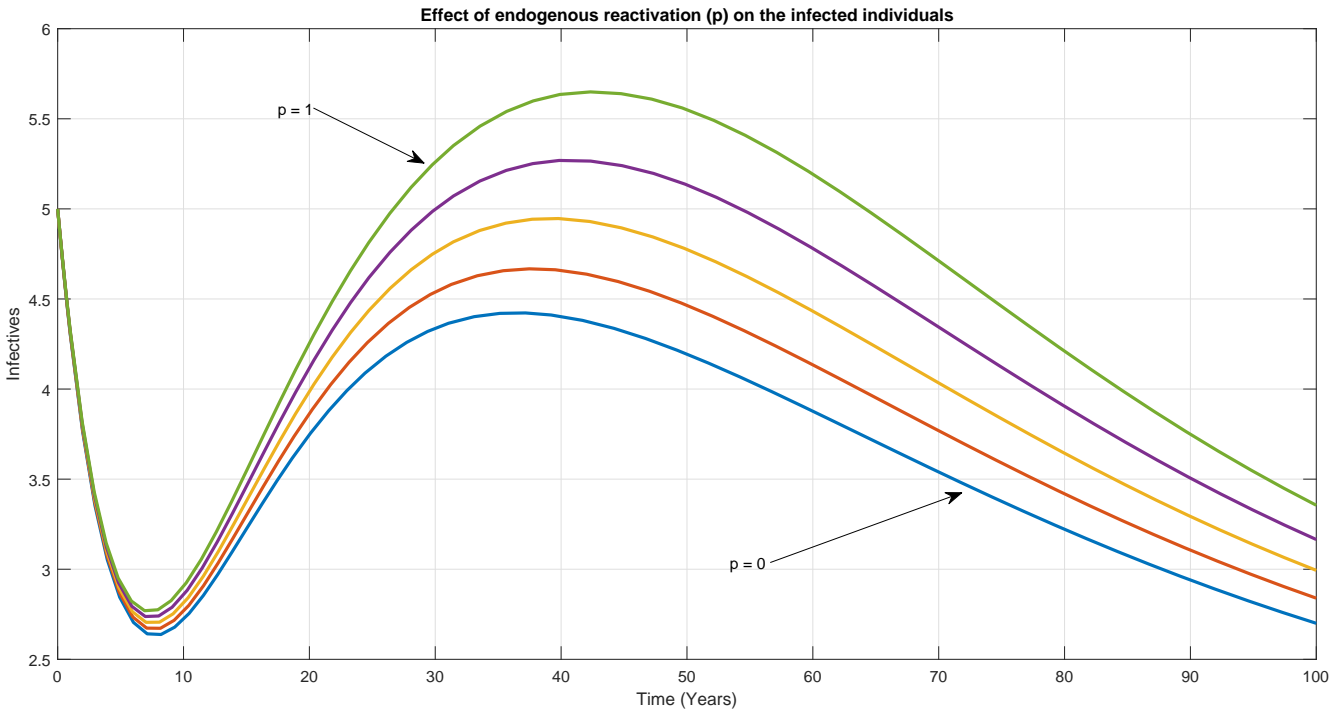

Figure 10. Effect of endogenous reactivation $p$ on the population of infected individuals when all other parameters are fixed: $\mu=0.15, \Lambda=5, \beta=1 \kappa=0.02, \delta=0.12, \sigma=0.02, \tau=2.3, \omega=0.2$, and $\theta=0.067$. 

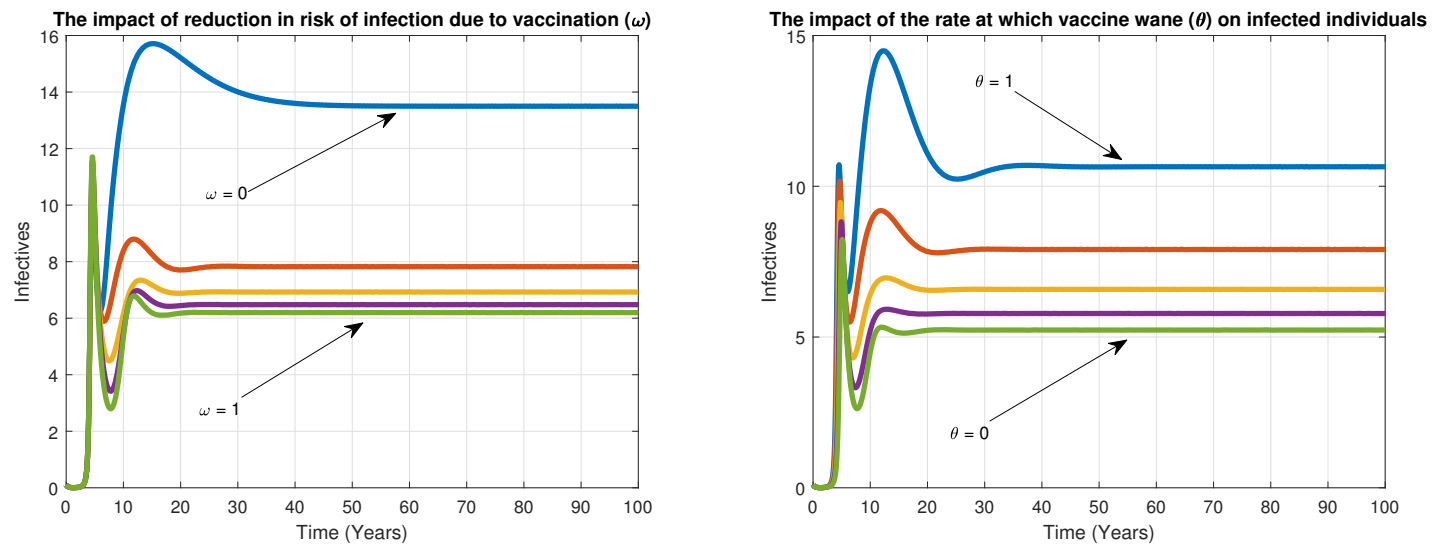

Figure 11. Impact of reduction in risk of infection due to vaccination $\omega$ (left) and the rate at which the vaccine wanes $\theta$ (right) on infected population using the same parameters as in Table 2, while $\omega$ and $\theta$ are varied.

\section{Conclusions}

The situation of an imperfect vaccine, accompanied by other exogenous factors (e.g., exogenous re-infection and re-infection among treated individuals), has been studied previously $[39,40]$. In this paper, we analyzed the impact of an imperfect vaccine and other exogenous factors on TB infection transmission dynamics, by constructing a deterministic numerical model to gain some insight into its dynamic behaviour. The effective reproduction number of the proposed system was determined using the next-generation matrix approach. In light of the employment of center manifold theory, a depth model analysis showed the existence of the backward bifurcation phenomenon, where a stable endemic equilibrium co-exists with a stable disease-free equilibrium under the condition of $R v<1$. Model dynamics were almost entirely dictated by the effective reproduction number, $R v$. The proposed model, consisting of five mutually exclusive compartments representing ТB dynamics, had a locally asymptotically stable disease-free equilibrium whenever a certain epidemiological threshold quantity, known as the effective reproduction number $(R v)$, was less than unity (i.e., $R v<1$ ). The model system (1) underwent backward bifurcation, where the disease may persist in the population, even if the classical epidemiological criterion of $R v<1$ is fulfilled; although it was still necessary to effectively control the spread of TB in a population. The initial population size entirely defined the condition (see Figure 4). A comparison between high and low vaccination coverage rates $\xi$ was likewise explored (see Figure 8). It was seen that the endemic equilibrium was higher in the lower case than in the high case.

For the examined SVEIRE model, an imperfect vaccine will reduce the disease burden and have a positive epidemiological effect on the population, even though its general effect increased with increased efficacy and coverage rate of the vaccines, as it has been realized that the utilization of an imperfect vaccine can — some of the time-bring about hindering results in the population $[84,85]$. A limited portion of individuals to be vaccinated at steadystate $\left(\eta_{c}\right)$, in order to achieve herd immunity, was established. Simulation of the system model (1) showed that using an imperfect vaccine can effectively control tuberculosis in a population. This is because the efficacy of the vaccine was remarkable high.

In summary, the key findings in this paper demonstrated that the TB model that considered an imperfect vaccine exhibited backward bifurcation. Our analysis results showed that there are two methods to prevent this phenomenon. First, reducing the effective reproductive number below the sub-threshold $R v c$ is required, in order to avoid the dangerous range $[R v c, 1]$. Second, the rate of vaccination should be increased in the range of the threshold value. Therefore, the vaccination rate plays a significant role.

Under the perspective of disease control campaigns, public policy-makers should consider the following two issues: First, attempt to keep away from the dangerous backward scenario, in which disease endemicity could persevere, even when the classic threshold 
value (i.e., the effective reproductive number) is less than unity. At this point, they may act by maintaining reasonably low vaccination of susceptible individuals. For this situation, the TB model with an imperfect vaccine suggests that disease control can tread the path of the classic strategy of decreasing the effective reproduction number $(R v c)$ below unity. Second, public policy-makers should be extremely careful if the backward scenario cannot be prevented, as merely keeping the effective reproductive number below unity can still contribute to the endemicity of the TB disease. In this scenario, the TB model suggested a need to reduce the effective reproductive number $(R v)$ below the $R v c$ sub-threshold. This objective can be achieved if $R v$ is reduced by organizing education programs that can influence the individual behavior of members of the public, which can act to appropriately decrease disease contact and, subsequently, future disease transmission.

In future work, the model can be modified to incorporate different dynamics that impact the spread of TB infection. In particular, some assumptions of the model can be relaxed or different measures can be incorporated, such that TB infection can be diminished or eliminated. Vaccination of children, sensitivity, optimal control, and cost-effectiveness analyses can also be carried out in order to glean more knowledge about the dynamics of the TB model.

Author Contributions: Conceptualization, F.S., F.A.A. and M.H.M.; methodology, F.S., F.A.A. and M.H.M.; software, F.S.; validation, F.S., F.A.A. and M.H.M.; formal analysis, F.S.; data curation, F.S., F.A.A. and M.H.M.; writing—original draft preparation, F.S.; writing-review and editing, F.S.; visualization, F.A.A.; supervision, F.A.A.; project administration, F.A.A.; funding acquisition, F.A.A. All authors have read and agreed to the published version of the manuscript.

Funding: This research was supported by Publication Fund Grant from the USM's Research Creativity and Management Office (RCMO) and School of Mathematical Sciences, Universiti Sains Malaysia.

Institutional Review Board Statement: Not applicable.

Informed Consent Statement: Not applicable.

Data Availability Statement: Not applicable.

Acknowledgments: The authors are gratefully acknowledge financial support from Publication Fund Grant from the USM's Research Creativity and Management Office (RCMO) and School of Mathematical Sciences, Universiti Sains Malaysia.

Conflicts of Interest: The authors declare no conflict of interest.

\section{References}

1. World Health Organization. Global Tuberculosis Report; WHO: Geneva, Switzerland, 2018; p. 214.

2. Khajanchi, S.; Das, D.K.; Kar, T.K. Dynamics of tuberculosis transmission with exogenous reinfections and endogenous reactivation. Phys. A 2018, 497, 52-71. [CrossRef]

3. Ullah, S.; Khan, M.A.; Farooq, M.; Gul, T. Modeling and analysis of Tuberculosis (TB) in Khyber Pakhtunkhwa, Pakistan. Math. Comput. Simul 2019, 165, 181-199. [CrossRef]

4. Bloom, B.R.; Murray, C.J. Tuberculosis: Commentary on a reemergent killer. Science 1992, 257, 1055-1064. [CrossRef]

5. Hill, A.N.; Becerra, J.E.; Castro, K.G. Modelling tuberculosis trends in the USA. Epidemiol. Infect. 2012, 257, 1862-1872. [CrossRef]

6. Abubakar, IDara, M.; Manissero, D.; Zumla, A. Tackling the spread of drug-resistant tuberculosis in Europe. Lancet 2012, 379, e21-e23. [CrossRef]

7. Behr, M.A. Tuberculosis Due to Multiple Strains: A Concern for the Patient? A Concern for Tuberculosis Control? Ann. Am. Thorac. Soc. 2004, 169, 554-555. [CrossRef]

8. Fatima, S.; Kumari, A.; Das, G.; Dwivedi, V.P. Tuberculosis vaccine: A journey from BCG to present. Life Sci. 2020, $252,117594$. [CrossRef]

9. Sudre, P.; Ten, Dam, G.; Kochi, A. Tuberculosis: A global overview of the situation today. Bull. World Health Org. 1992, 70, 149.

10. Dolin, P.J.; Raviglione, M.C.; Kochi, A. Global tuberculosis incidence and mortality during 1990-2000. Bull. World Health Org. 1994, 72, 213.

11. Moghadas, S.M.; Alexander, M.E. Exogenous reinfection and resurgence of tuberculosis: A theoretical framework. J. Biol. Syst. 2004, 12, 231-247. [CrossRef]

12. Castillo-Chavez, C.; Song, B. Dynamical models of tuberculosis and their applications. Math. Biosci. Eng. 2004, 1, 361. [CrossRef] [PubMed] 
13. Adebiyi, A.O. Mathematical Modeling of the Population Dynamics of Tuberculosis; University of the Western Cape: Cape Town, South Africa, 2016.

14. Richardson, M.; Carroll, N.M.; Engelke, E.; van der Spuy, G.D.; Salker, F.; Munch, Z.; van Helden, P.D. Multiple Mycobacterium tuberculosis strains in early cultures from patients in a high-incidence community setting. J. Clin. Microbiol. 2002, 40, 2750-2754. [CrossRef] [PubMed]

15. Reichman, L.B.; Hershfield, E.S. Tuberculosis: A Comprehensive International Approach; CRC Press: Boca Raton, FL, USA, 2000.

16. Lin, P.L.; Ford, C.B.; Coleman, M.T.; Myers, A.J.; Gawande, R.; Ioerger, T.; Flynn, J.L. Sterilization of granulomas is common in active and latent tuberculosis despite within-host variability in bacterial killing. Nat. Med. 2014, 20, 75-79. [CrossRef] [PubMed]

17. Smith, P.G.; Moss, A.R. Epidemiology of tuberculosis. In Tuberculosis; American Society for Microbiology: Washington, DC, USA, 1994; pp. 47-59.

18. Blower, S.M.; Mclean, A.R.; Porco, T.C.; Small, P.M.; Hopewell, P.C.; Sanchez, M.A.; Moss, A.R. The intrinsic transmission dynamics of tuberculosis epidemics. Nat. Med. 1995, 1, 815-821. [CrossRef] [PubMed]

19. Vynnycky, E.; Fine, P.E. Lifetime risks, incubation period, and serial interval of tuberculosis. Am. J. Epidemiol. 2000, 152, 247-263. [CrossRef]

20. Drain, P.K.; Bajema, K.L.; Dowdy, D.; Dheda, K.; Naidoo, K.; Schumacher, S.G.; Sherman, D.R. Incipient and subclinical tuberculosis: A clinical review of early stages and progression of infection. Clin. Microbiol. Rev. 2018, 31. [CrossRef]

21. Kar, T.K.; Mondal, P.K. Global dynamics of a tuberculosis epidemic model and the influence of backward bifurcation. J. Math. Model. Algorithms 2012, 11, 433-459. [CrossRef]

22. Wangari, I.M.; Davis, S.; Stone, L. Backward bifurcation in epidemic models: Problems arising with aggregated bifurcation parameters. Appl. Math. Model. 2016, 40, 1669-1675. [CrossRef]

23. Cohen, T.; Colijn, C.; Finklea, B.; Murray, M. Exogenous re-infection and the dynamics of tuberculosis epidemics: Local effects in a network model of transmission. J. R. Soc. Interface 2007, 4, 523-531. [CrossRef]

24. Gomes, M.G.M.; Rodrigues, P.; Hilker, F.M.; Mantilla-Beniers, N.B.; Muehlen, M.; Paulo, A.C.; Medley, G.F. Implications of partial immunity on the prospects for tuberculosis control by post-exposure interventions. J. Theor. Biol. 2007, 248, 608-617. [CrossRef]

25. Liu, X.; Takeuchi, Y.; Iwami, S. SVIR epidemic models with vaccination strategies. J. Theor. Biol. 2008, 253, 1-11. [CrossRef] [PubMed]

26. Anderson, R.M.; Anderson, B.; Robert, M.M. Infectious Diseases of Humans: Dynamics and Control; Oxford University Press: Oxford, UK, 1992.

27. Buonomo, B.; Lacitignola, D. On the backward bifurcation of a vaccination model with nonlinear incidence. Nonlinear Anal. Model. Control 2011, 16, 30-46. [CrossRef]

28. Buonomo, B.; Della Marca, R. Oscillations and hysteresis in an epidemic model with information-dependent imperfect vaccination. Math. Comput. Simul. 2019, 162, 97-114. [CrossRef]

29. Andersen, P.; Doherty, T.M. The success and failure of BCG-Implications for a novel tuberculosis vaccine. Nat. Rev. Microbiol. 2005, 3, 656-662. [CrossRef] [PubMed]

30. Nadolinskaia, N.I.; Karpov, D.S.; Goncharenko, A.V. Vaccines against Tuberculosis: Problems and Prospects. Appl. Biochem. Microbiol. 2020, 56, 497-504. [CrossRef]

31. Fine, P.E.; Carneiro, I.A.; Milstien, J.B.; Clements, C.J. Issues Relating to the Use of BCG in Immunization Programmes: A Discussion Document (No. WHO/V and B/99.23); World Health Organization; Geneva, Switzerland, 1999.

32. van Rie, A.; Warren, R.; Richardson, M.; Victor, T.C.; Gie, R.P.; Enarson, D.A.; van Helden, P.D. Exogenous reinfection as a cause of recurrent tuberculosis after curative treatment. N. Engl. J. Med. 1999, 341, 1174-1179. [CrossRef]

33. Bhunu, C.P.; Garira, W.; Mukandavire, Z.; Zimba, M. Tuberculosis transmission model with chemoprophylaxis and treatment. Bull. Math. Biol. $2008,70,1163-1191$.

34. Feng, Z.; Castillo-Chavez, C.; Capurro, A.F. A model for tuberculosis with exogenous reinfection. Theor. Popul. Biol. 2000 57, 235-247. [CrossRef]

35. Song, B.; Castillo-Chavez, C.; Aparicio, J.P. Tuberculosis models with fast and slow dynamics: The role of close and casual contacts. Math. Biosci. 2002, 180, 187-205.

36. Liu, S., Li, Y., Bi, Y., and Huang, Q. Mixed vaccination strategy for the control of tuberculosis: A case study in China. Math. Biosci. Eng. 2017, 14, 695.

37. Renardy, M.; Kirschner, D.E. Evaluating vaccination strategies for tuberculosis in endemic and non-endemic settings. J. Theor. Biol 2019, 469, 1-11. [CrossRef]

38. Gerberry, D.J. Practical aspects of backward bifurcation in a mathematical model for tuberculosis. J. Theor. Biol. 2016, 388, 15-36. [CrossRef]

39. Nkamba, L.N.; Manga, T.T.; Agouanet, F.; Mann Manyombe, M.L. Mathematical model to assess vaccination and effective contact rate impact in the spread of tuberculosis. J. Biol. Dyn. 2019, 13, 26-42. [CrossRef]

40. Egonmwan, A.O.; Okuonghae, D. Mathematical analysis of a tuberculosis model with imperfect vaccine. Int. J. Biomath. 2019, 12, 1950073. [CrossRef]

41. Wangari, I. Backward Bifurcation and Reinfection in Mathematical Models of Tuberculosis. Ph.D. Thesis, RMIT University, Melbourne, Australia, 2017. 
42. Huang, W.; Cooke, K.L.; Castillo-Chavez, C. Stability and bifurcation for a multiple-group model for the dynamics of HIV/AIDS transmission. SIAM J. Appl. Math. 1992, 52, 835-854. [CrossRef]

43. Hadeler, K.P.;Van den Driessche, P. Backward bifurcation in epidemic control. Math. Biosci. 1997, 146, 15-35. [CrossRef]

44. Nudee, K.; Chinviriyasit, S.; Chinviriyasit, W. The effect of backward bifurcation in controlling measles transmission by vaccination. Chaos Soliton Fract. 2019, 123, 400-412. [CrossRef]

45. Nadim, S.S.; Chattopadhyay, J. Occurrence of backward bifurcation and prediction of disease transmission with imperfect lockdown: A case study on COVID-19. Chaos Soliton Fract. 2019, 140, 110163. [CrossRef]

46. Greenhalgh, D.; Diekmann, O.; de Jong, M.C. Subcritical endemic steady states in mathematical models for animal infections with incomplete immunity. Math. Biosci. 2000, 165, 1-25. [CrossRef]

47. Gumel, A.B. Causes of backward bifurcations in some epidemiological models. J. Math. Anal. Appl. 2012, 395, 355-365.

48. Hadeler, K.P.; Castillo-Chávez, C. A core group model for disease transmission. Math. Biosci. 1995, 128, 41-55. [CrossRef]

49. Dushoff, J. Incorporating immunological ideas in epidemiological models. J. Theor. Biol. 1996, 180, 181-187. [CrossRef]

50. Feng, Z.; Velasco-Hernandez, J.Tapia-Santos, B. A mathematical model for coupling within-host and between-host dynamics in an environmentally-driven infectious disease. Math. Biosci. 2013, 241, 49-55. [CrossRef]

51. Wang, W. Backward bifurcation of an epidemic model with treatment. Math. Biosci. 2006, 201, 58-71. [CrossRef]

52. Cui, J.; Mu, X.; Wan, H. Saturation recovery leads to multiple endemic equilibria and backward bifurcation. J. Theor. Biol. 2008, 254, 275-283. [CrossRef]

53. Hu, Z.; Ma, W.; Ruan, S. Analysis of SIR epidemic models with nonlinear incidence rate and treatment. Math. Biosci. 2013, 238, 12-20. [CrossRef]

54. Dushoff, J.; Huang, W.; Castillo-Chavez, C. Backwards bifurcations and catastrophe in simple models of fatal diseases. J. Math. Biol. 1998, 36, 227-248. [CrossRef]

55. Buonomo, B.; Vargas-De-León, C. Stability and bifurcation analysis of a vector-bias model of malaria transmission. Math. Biosci. 2013, 242, 59-67. [CrossRef]

56. Gómez-Acevedo, H.; Li, M.Y. Backward bifurcation in a model for HTLV-I infection of CD4+ T cells. Bull. Math. Biol. 2005, 67, 101-114. [CrossRef]

57. Martcheva, M.; Thieme, H.R. Progression age enhanced backward bifurcation in an epidemic model with super-infection. J. Math. Biol. 1998, 46, 385-424. [CrossRef]

58. Omame, A.; Okuonghae, D.; Umana, R.A.; Inyama, S.C. Analysis of a co-infection model for HPV-TB. Appl. Math. Model. 2020, 77, 881-901. [CrossRef]

59. Magpantay, F.M.; Riolo, M.A.; De Celles, M.D.; King, A.A.; Rohani, P. Epidemiological consequences of imperfect vaccines for immunizing infections. SIAM J. Appl. Math. 2014, 74, 1810-1830. [CrossRef] [PubMed]

60. Gumel, A.B.; McCluskey, C.C.; Watmough, J. An SVEIR model for assessing potential impact of an imperfect anti-SARS vaccine. Math. Biosci. Eng. 2006, 3, 485. [PubMed]

61. Moghadas, S. M. Modelling the effect of imperfect vaccines on disease epidemiology. Discrete. Continuous. Dyn. Syst. Ser. B 2004, 4, 999-1012. [CrossRef]

62. Cai, L.M.; Li, Z.; Song, X. Global analysis of an epidemic model with vaccination. J. Math. Comput. 2018, 57, 605-628. [CrossRef]

63. Obasi, C.; Mbah, G.C.E. On the stability analysis of a mathematical model of Lassa fever disease dynamics. J. Nig. Soc. Math. Biol. 2019, 2, 135-144.

64. Egonmwan, A.O.; Okuonghae, D. Analysis of a mathematical model for tuberculosis with diagnosis. J. Appl. Math. Comput. 2019, 59, 129-162.

65. Adeniyi, M.O.; Ekum, M.I.; Iluno, C.; Oke, S.I. Dynamic model of COVID-19 disease with exploratory data analysis. Sci. Afr. 2020, 9, e00477.

66. Oke, S.I.; Ojo, M.M.; Adeniyi, M.O.; Matadi, M.B. Mathematical modeling of malaria disease with control strategy. Commun. Math. Biol. Neurosci. 2020, 2020, 43.

67. Birkhoff, G.; Rota, G.C. Ordinary Differential Equations; Wiley: Hoboken, NJ, USA,1989.

68. Hethcote, H.W. The mathematics of infectious diseases. SIAM Rev. 2000, 42, 599-653. [CrossRef]

69. Diekmann O.; Heesterbeek J.A.P.; Metz J.A. On the definition and the computation of the basic reproduction ratio $R_{0}$ in models for infectious diseases in heterogeneous populations. J. Math. Biol. 1990, 28, 365-382. [CrossRef] [PubMed]

70. Dietz, K. The estimation of the basic reproduction number for infectious diseases. Stat. Methods Med. Res. 1993, 2, $23-41$. [CrossRef] [PubMed]

71. Van den Driessche, P.; Watmough J. Further Notes on the Basic Reproduction Number in Mathematical Epidemiology; Springer: Berlin/Heidelberg, Germany, 2008; pp. 159-178.

72. Garba, S.M. Mathematical Modeling and Analysis of Dengue Transmission Dynamics. Ph.D. Thesis, Universiti Putra Malaysia, Seri Kembangan, Malaysia, 2008.

73. Salle, J.L. Stability by Liapunov's Direct Method with Applications; Academic Press: Cambridge, MA, USA, 1961.

74. Arino, J.; McCluskey, C.C.; van den Driessche, P. Global results for an epidemic model with vaccination that exhibits backward bifurcation. SIAM J. Appl. Math. 2003, 64, 260-276. [CrossRef]

75. Colditz, G.A.; Brewer, T.F.; Berkey, C.S.; Wilson, M.E.; Burdick, E.; Fineberg, H.V.; Mosteller, F. Efficacy of BCG vaccine in the prevention of tuberculosis: meta-analysis of the published literature. JAMA 1994, 271, 698-702. [CrossRef] 
76. Aronson, N.E.; Santosham, M.; Comstock, G.W.; Howard, R.S.; Moulton, L.H.; Rhoades, E.R.; Harrison, L.H. Long-term efficacy of BCG vaccine in American Indians and Alaska Natives: A 60-year follow-up study. JAMA 2004, 291, 2086-2091. [CrossRef]

77. Sterne, J.A.C.; Rodrigues, L.C.; Guedes, I.N. Does the efficacy of BCG decline with time since vaccination. Int. J. Tuberc. Lung Dis. 1998, 2, 200-207.

78. Nguipdop-Djomo, P.; Heldal, E.; Rodrigues, L.C.; Abubakar, I.; Mangtani, P. Duration of BCG protection against tuberculosis and change in effectiveness with time since vaccination in Norway: A retrospective population-based cohort study. Lancet Infect. Dis. 2016, 16, 219-226. [CrossRef]

79. Alexander, M.E.; Moghadas, S.M.; Rohani, P.; Summers, A.R. Modelling the effect of a booster vaccination on disease epidemiology. J. Math. Biol. 2006, 52, 290-306.

80. Gomes, M.G.M.; Franco, A.O.; Gomes, M.C.; Medley, G.F. The reinfection threshold promotes variability in tuberculosis epidemiology and vaccine efficacy. Proc. R. Soc. Lond. Ser. B J. Biol. Sci. 2004, 271, 617-623. [CrossRef]

81. Porco, T.C.; Blower, S.M. Quantifying the intrinsic transmission dynamics of tuberculosis. Theor. Popul. Biol. 1998, 54, 117-132.

82. Adewale, S. O., Podder, C. N., Gumel, A. B. Mathematical analysis of a TB transmission model with DOTS. Can. Appl. Math. Q. 2009, 17, 1-36.

83. Gomes, M.G.M.; Aguas, R.; Lopes, J.S.; Nunes, M.C.; Rebelo, C.; Rodrigues, P.; Struchiner, C.J. How host heterogeneity governs tuberculosis reinfection? Proc. Roy. Soc. B-Biol. Sci. 2012, 279, 2473-2478. [CrossRef] [PubMed]

84. Elbasha, E.H.; Gumel, A.B. Theoretical assessment of public health impact of imperfect prophylactic HIV-1 vaccines with therapeutic benefits. Bull. Math. Biol. 2006, 68, 577. [CrossRef] [PubMed]

85. Gandon, S.; Mackinnon, M.; Nee, S.; Read, A. Imperfect vaccination: some epidemiological and evolutionary consequences. Proc. R. Soc. B-Biol. Sci. 2003, 270, 1129-1136. [CrossRef] [PubMed] 